\title{
Global Phosphoproteome Analysis Reveals Significant Differences Between Sporulated Oocysts of Virulent and Avirulent Strains of Toxoplasma Gondii
}

\section{Ze-Xiang Wang ( $\nabla$ neau601@126.com )}

College of Veterinary Medicine, Gansu Agricultural University, Lanzhou, Gansu Province 730070, PR China

Rui-Si Hu

College of Animal Science and Technology, Jilin Agricultural University

Xing-Quan Zhu

Lanzhou Veterinary Research Institute

Xiao-Lin Sun

Gansu Agricultural University

Hany M. Elsheikha

University of Nottingham

\section{Research}

Keywords: Toxoplasma gondii, Genotype, Sporulated oocyst, IBT, Phosphoproteomics

Posted Date: October 2nd, 2020

DOI: https://doi.org/10.21203/rs.3.rs-84449/v1

License: (c) (1) This work is licensed under a Creative Commons Attribution 4.0 International License.

Read Full License

Version of Record: A version of this preprint was published at Microbial Pathogenesis on October 1st, 2021. See the published version at https://doi.org/10.1016/j.micpath.2021.105240. 


\section{Abstract}

Background: The apicomplexan protozoan parasite Toxoplasma gondii is one of the most successful intracellular parasites capable of infecting warm-blooded animals. Several strains of $T$. gondii have been described and typed as virulent or avirulent based on their pathogenicity in mice, as well as sporulated oocysts of strains belonging to distinct $T$. gondii genotypes. Phosphorylation is a reversable form of protein post-translational modification (PTM) that influences many biological processes and is used by some apicomplexan parasites to facilitate manipulation of the host cells. Phosphoproteomic analysis of oocysts of $T$. gondii strains belonging to distinct genotypes may reveal mechanisms contributing to the differences in the virulence of this parasite at the posttranslational level.

Methods: In this study, the differences in the phosphoproteomic landscape of sporulated oocysts between virulent and avirulent strains of Toxoplasma gondii were examined using a global phosphoproteomics approach. Phosphopeptides from sporulated oocysts of the virulent PYS strain (Chinese ToxoDB\#9) and the avirulent PRU strain (type II) were enriched by titanium dioxide $\left(\mathrm{TiO}_{2}\right)$ affinity chromatography and quantified using isobaric tag (iBT) approach. Motif analysis, GO enrichment, KEGG pathway analysis, STRING analysis and kinase related network analysis of phosphopeptides were conducted to discover distinct difference in sporulated oocysts between virulent and avirulent $T$. gondii strains.

Results: A total of 10,645 unique phosphopeptides, 8,181 nonredundant phosphorylation sites and 2,792 phosphoproteins were identified. We also detected 4,129 differentially expressed phosphopeptides (DEPs) between sporulated oocysts of PYS strain and PRU strain ( $\mid \log 1.5$ fold changel $>1$ and $p<0.05$ ), including 2,485 upregulated and 1,644 downregulated phosphopeptides. Motif analysis identified 24 motifs from the upregulated phosphorylated peptides including 22 serine motifs and two threonine motifs (TPE and TP), and 15 motifs from the downregulated phosphorylated peptides including 12 serine motifs and three threonine motifs (TP, RxxT and KxxT) in PYS strain when comparing PYS strain to PRU strain. Several kinases were consistent with motifs of overrepresented phosphopeptides, such as PKA, PKG, CKII, IKK, MAPK, EGFR, INSR, Jak, Syk, Src, Ab1. GO enrichment, KEGG pathway analysis and STRING analysis revealed DEPs significantly enriched in many biological processes and pathways. Kinase related network analysis showed that AGC kinase had the greatest connected peptides.

Conclusions: The present study revealed the global phosphoproteomic differences in sporulated oocysts between virulent and avirulent $T$. gondii strains of different genotypes. Abundant phosphopeptides in sporulated oocysts between virulent and avirulent $T$. gondii strains exhibited distinct difference in the conserved motifs, GO terms, enriched pathways and PPI networks. The kinase associated network analysis indicated the role of phosphorylation in the transformation of $T$. gondii kinases. AGC kinase was the most connected kinases peptides. These data provide new insight into the phenotypic differences in sporulated oocysts of virulent and avirulent $T$. gondii strains.

\section{Background}


The apicomplexan protozoan parasite Toxoplasma gondii is one of the most successful intracellular parasites capable of infecting warm-blooded animals [1]. Approximately one-third of the world human population have been estimated to be infected by this parasite [2]. Although most $T$. gondii infections in immuno-competent individuals are asymptomatic, severe or even fatal clinical illness can occur in immuno-compromised patients [3]. Infection with $T$. gondii during pregnancy can result in abortion and stillbirth, or hydrocephalus and retinochoroiditis in the survived neonates [4]. Several strains of T. gondii have been described and typed as virulent or avirulent based on their pathogenicity in mice [3, 5-6]. Significant phenotypic variations also exist between different strains of the most prevalent genotype Chinese 1 (ToxoDB\#9) in animals and humans in China [7]. Despite the significant differences in virulence among strains of different $T$. gondii genotypes, the genetic difference deleted among these strains was only around $1 \%[8-9]$.

Understanding the molecular basis of the virulence mechanisms among the different genotypes of $T$. gondii can identify novel contributors to the significant genotype-related differences in the parasite's virulence. Proteomics approach revealed virulence-related genotypic differences, where strain-specific proteomic signatures were detected in the sporulated oocysts of virulent and avirulent strains belonging to distinct $T$. gondii genotypes [10]. Also, iTRAQ technology combined with titanium dioxide affinity chromatography revealed strain-specific variations in the phosphoprotein profile between tachyzoites of different $T$. gondii genotypes [11]. Phosphorylation is a reversable form of protein post-translational modification (PTM) that influences many biological processes and is used by some apicomplexan parasites to facilitate manipulation of the host cells [12-13]. Therefore, understanding the phosphoproteomic profiles of sporulated oocysts between virulent and avirulent strains of $T$. gondii is also essential because it enhances the understanding of the underlying molecular mechanisms contributing to $T$. gondii virulence.

In the present study, a global phosphoproteomics approach was used to analyze the phosphoproteomes of the virulent ToxoDB\#9 (PYS) strain and the avirulent ToxoDB\#1 (PRU) strain. We tested the hypothesis that phosphoproteomic analysis of oocysts of $T$. gondii strains belonging to distinct genotypes may reveal mechanisms contributing to the differences in the virulence of this parasite at the posttranslational level. Our data showed many differences in the phosphoproteomes of sporulated oocysts of two distinct T. gondii genotypes.

\section{Methods}

\section{Production, Purification and Sporulation of Oocysts}

The tachyzoites of the virulent PYS (ToxoDB\#9 genotype) strain and the cysts of avirulent Prugniuad (PRU; ToxoDB\#1) strain were maintained in mice in our laboratory. $10^{5}$ tachyzoites of PYS strain were intraperitoneally injected into 16-week-old female guinea pigs. One month later, guinea pigs were euthanized, and a specific-pathogen-free (SPF) kitten was inoculated with homogenate of the brain and muscle of guinea pigs. The kitten was examined by modified agglutination test (MAT) prior to the 
inoculation in order to confirm that they aren't infected with T. gondii. After the infection, the kitten's feces were gathered daily and tested by a light microscope (Japan, Olumpus) for detection of the oocysts. For the PRU strain, cysts were routinely passaged via oral inoculation in six-week-old mice. Specific-pathogenfree mice was infected with 200 cysts through oral route and its feces was tested to detect the shedding of oocysts of the PRU strain as mentioned above.

Once oocysts of PYS strain and PRU strain were detected, they were isolated and purified from the kitten's feces by using caesium chloride $(\mathrm{CsCl})$ centrifugation method [14]. Firstly, cat feces were blended with water and the fecal suspension was filtered using a $250-\mu \mathrm{m}$ pore size tea strainer. Afterward, the filtrates were centrifugated (60 $\mathrm{g}$ for $15 \mathrm{mins}$ ) and the sediment was rinsed three times with phosphate-buffered saline (PBS). Following the final wash, the supernatant was clarified, the pellet was suspended in 5 volumes of sucrose solution (1.15 specific gravity) and centrifugated for 10 mins at $350 \mathrm{~g}$ in order to concentrate oocysts. The oocysts were collected from the supernatant, blended in TE buffer (10 mM Tris$\mathrm{HCl}, 1 \mathrm{mM}$ EDTA, $\mathrm{pH}=8.0$ ) and purified applying a discontinuous $\mathrm{CsCl}$ density gradient method as previously described [15-16]. Finally, oocysts within the opaque-to-white layer were harvested and washed twice with $0.85 \%$ saline followed by resuspension in PBS and storage at $4{ }^{\circ} \mathrm{C}$. For sporulation, the suspension of oocysts was centrifugated at $350 \mathrm{~g}$ and the pelleted oocysts were resuspended in $2 \%$ $\mathrm{H}_{2} \mathrm{SO}_{4}$ (sulfuric acid) to induce sporulation on a shaker in an aerobic condition for 7 days at ambient temperature. After two washes in PBS, the sporulated oocysts were suspended in sulfuric acid and stored at $4^{\circ} \mathrm{C}$ until use. Prior to use, sulfuric acid in sporulated oocysts suspension were removed by three washes in PBS and sporulated oocysts were ultimately resuspended in PBS.

\section{Extraction and Digestion of Protein}

Total protein was extracted from at least three biological replicates of mature oocysts of PYS strain and PRU strain ( $\left(10^{7}\right.$ per each biological replicate). The sporulated oocyst's suspension was centrifugated at $350 \mathrm{~g}$ for $15 \mathrm{~min}$ followed by suspension of the pelleted oocysts in lysis buffer $(20 \mathrm{mM} \mathrm{Tris}-\mathrm{HCl}, 0.2 \%$ SDS, $7 \mathrm{M}$ urea/2 M thiourea, $10 \mathrm{mM}$ DTT, pH 8.5) supplemented with phosphatase inhibitors (PhosSTOP, Roche) and phenylmethylsulfonyl (PMSF, $1 \mathrm{mM}$, Thermo Scientific). The sporulated oocysts within the suspension were lysed by sonication on ice ( $2 \mathrm{sec}$ ON/3 sec OFF pulses for $5 \mathrm{~min}$ ) and the suspension was pelleted $\left(25,000 \mathrm{~g}\right.$ for $20 \mathrm{~min}$ at $\left.4^{\circ} \mathrm{C}\right)$ to eliminate lysate debris. Subsequently, the supernatant was deoxidized and alkylated by dithiothreitol (DTT, $10 \mathrm{mM}, 60 \mathrm{~min}$ at $\left.56^{\circ} \mathrm{C}\right)$ and iodoacetamide $(55 \mathrm{mM}$ IAM for 45 min at ambient condition), respectively. The Bradford assay was used to measure the protein concentration. To digest the protein extracted from each biological replicate/sample $300 \mu \mathrm{g}$ trypsin (Promega) were added at enzyme-to-protein ratio of $1: 40$ at $37^{\circ} \mathrm{C}$ for $24 \mathrm{hr}$. The enzymatic reaction was terminated by adding formic acid to a final concentration of $0.5 \%(\mathrm{v} / \mathrm{v})$. Finally, samples were desalted using Strata $X$ solid-phase extraction columns (Phenomenex) and dried utilizing a SpeedVac, before being used for iBT labeling.

\section{iBT Labeling}


The dried peptides were labeled using 6-plexed iBT reagent (BGI-Shenzhen, Shenzhen, China) as per the manufacturer's instructions. Briefly, the peptides were mixed with labeling reagent blended in $50 \mu \mathrm{l}$ isopropyl alcohol followed by an incubation at ambient temperature for $2 \mathrm{hr}$. Then, a Sep-Pack $\mathrm{C}_{18}$ Cartridges (Waters) was used to desalt and concentrate the labelled peptides. The labelled peptides were stored at $-80^{\circ} \mathrm{C}$ until used for enrichment of phosphopeptides. Proteins of sporulated oocysts of PYS and PRU strains were labelled with iBT tags as follows: PYS strain $(114,115 \mathrm{~N}$ and $115 \mathrm{C})$ and PRU strain $(116 \mathrm{~N}, 116 \mathrm{C}$ and 117$)$.

\section{Enrichment of Phosphopeptides Utilizing $\mathrm{TiO}_{2}$ Beads}

$\mathrm{TiO}_{2}$ (Titanium dioxide, GL Sciences, Tokyo, Japan) beads were used to enrich the labelled phosphopeptides. Before enrichment, $1 \mathrm{ml}$ loading buffer (65\% ACN / $2 \%$ glutamic acid / $2 \%$ TFA) was used to suspend $\mathrm{TiO}_{2}$ beads with peptides-to-beads ratio of 1:4 for $10 \mathrm{~min}$. Afterwards, the labeled peptides were mixed with the $\mathrm{TiO}_{2}$ suspension and the mixture was agitated on a rotator for $1 \mathrm{hr}$ at $37^{\circ} \mathrm{C}$. Then, the mixture was centrifugated at $12,000 \mathrm{~g}$ for $5 \mathrm{~min}$, and the sediment was rinsed three times with 2 $\mathrm{ml}$ loading buffer $(12,000 \mathrm{~g}$ for $5 \mathrm{~min})$. After the final wash, the pellet was dissolved with $600 \mu \mathrm{l}$ elution buffer and the mixture was agitated for 20 min succeeded by a pellet at $12,000 \mathrm{~g}$ for $1 \mathrm{~min}$. The pellet was rinsed with $500 \mu$ l elution buffer using the same agitation and centrifugation conditions mentioned above. Finally, the supernatant from the both centrifugations, which contained phosphopeptides enriched by $\mathrm{TiO}_{2}$, were collected and vacuum-dried for LC-MS/MS analysis.

\section{LC-MS/MS}

Utilizing a High pH Reversed-Phase Peptide Fractionation Kit (Thermo Scientific Pierce), the samples' fractions were isolated according to the manufacturer's recommendation. Initially, $300 \mu \mathrm{l} 0.1 \%$ TFA was added to the samples to dissolve the enriched peptides, which were laded into the equilibrated fractionation spin column. After enriched peptides binding to the resin, the column was washed with sterile water by centrifugation at low speed in order to desalt the enrich peptides. Then, the bound peptides were eluted from the spin columns and harvested into six different fractions by a volatile high$\mathrm{pH}$ elution which included a gradient of accelerative acetonitrile concentrations. Finally, fractions were respectively collected and vacuum-dried by centrifugation. After fractionation, the $\mathrm{Q}$ Exactive Mass Spectrometer (Thermal Scientific) conjugated with HPLC (Phenomenex columns Gemini-NX 3u C18 110A $150 * 2.00 \mathrm{~mm}$ ) was used to perform the LC-MS/MS analysis. Samples dissolved in $20 \mu \mathrm{l}$ buffer A ( $2 \%$ $\mathrm{ACN}, 0.1 \% \mathrm{FA}$ ) were laded into a C18 trap column at a speed of $8 \mu \mathrm{l} / \mathrm{min}$ for $4 \mathrm{~min}$. The samples were eluted by a linear gradient of buffer $B(95 \% \mathrm{ACN}, 0.1 \% \mathrm{FA})$, which was running at $300 \mathrm{nl} / \mathrm{min}$ and commenced from $5 \%$ buffer $B$, and then raised into $35 \%$ buffer $B$ within 45 min. Within 5 min, the linearizing gradient of buffer B gradually increased into $60 \%$, followed by an increase into $80 \%$ buffer B in 2 min. After a retention of $80 \%$ buffer B for 2 min, the concentration of buffer B decreased into $5 \%$ within $1 \mathrm{~min}$ and $5 \%$ buffer $B$ was kept for $10 \mathrm{~min}$. The spectra of first-grade MS involved in the following parameter: scan scope $=350$ to $1500 \mathrm{~m} / \mathrm{z}$; resolution $=70,000$; maximum injection time $=40 \mathrm{~ms}$; AGC target $=3 e 6$. As for the second grade MS, spectra were obtained during in the resolution of 17,500 with 40 
ms maximum injection time and maximum injection time were 1e5, in which the 20 top precursors were screened out in the mode of HCD (high-energy collisional dissociation).

\section{Database Search}

The Proteome Discoverer 1.4 (Thermo Fisher Scientific) combined with a Mascot search engine (version 2.3, Matrix Science) was used to search T. gondii database

(http://www.toxodb.org/common/downloads/release-10.0/Toxoplasma gondii ME49/fasta/data/) and identify phosphopeptides. Parameters used in the search were set as follow: enzyme = trypsin; peptide mass tolerance $=20 \mathrm{ppm}$; fragment mass tolerance $=0.05 \mathrm{Da}$; fixed modification $=$ IBT-10plex (N-term), carbamidomethyl (C), IBT-8plex (K); variable modification = oxidation (M), acetyl (protein N-term), deamidated (NQ); phosphorylation (S/T/Y) maximum missed cleavage $=2$. In order to filter the results, $p$ value was set as $p \leq 0.05$ in order to identify and quantify phosphopeptides, and phosphosites with a phosphoRS probability not less than 0.75 were deemed as reliable phosphosites. With regard to differentially expressed phosphopeptides (DEPs), those with a fold-change $>1.5$ or $<0.75$ were determined as upregulated or downregulated DEPs, respectively. Hierarchical clustering method was applied to group the DEPs and heat maps were created by the software Multiple Experiment Viewer (Mev, version 4.9.0).

\section{Bioinformatic Analysis}

The online software Motif-X (http://motif-x.med.harvard.edu/) was applied to search the phosphorylation motifs of phosphopeptides in the present study. Phosphopeptides with the length of \pm 7 amino acids neighboring the phosphorylation site were analyzed using the Motif- $\mathrm{X}$ software for searching conserved motifs adjacent to phosphorylation sites. Motifs identified were graphically shown with logo-like representation. The functions of the DEPs were sorted into molecular function (MF), biological process (BP) and cell component (CC) using Gene Ontology (GO) analysis (http://www.geneontology.org). KEGG (Kyoto Encyclopedia of Genes and Genomes) analysis was conducted to annotate the DEPs to pathways present in the KEGG database (http://www.genome.jp/kegg/). The significantly enriched pathways were displayed as bubble charts. STRING (Search Tool for the Retrieval of Interacting Genes/Protein, http://string-db.org/) database was used to survey processes affected by phosphorylation and to study interaction networks of the DEPs participate in.

Interactors whose confidence were higher (combined score $\geq 0.7$ ) were displayed in protein-protein interaction (PPI) networks exhibited by Cytoscape (version 3.0.2).

\section{Correlation between Phosphopeptides and T. gondii Kinase Peptides}

The HMMER (http://hmmer.org) was used to search the Pfam 31.0 database with the purpose of identifying sequences of the phosphorylated proteins that are related to kinases in the Pfam 31.0 database [17]. An array of phosphorylated peptides matched to protein kinase and protein kinase domains was obtained. Afterward, Pearson correlational analysis was performed to connect kinasematched phosphorylated peptides with total phosphorylated peptides in our dataset. This analysis was 
conducted based on the DEPs ratio values per each strain. Only phosphorylated peptide with correlations $>0.998$ were selected. DEPs with $\log _{1.5}$ fold change values $-0.75{ }^{3} \mathrm{RS} \leq 1.50$ were excluded. Cytoscape 3.6.0 (https://cytoscape.org) was used to visualize the network.

\section{Prediction of Protein Interactions Enabled or Disabled by Phosphorylation}

To characterize promising protein interactions enabled or disabled by phosphorylation, Mechismo [18] was used to predict phospho-enabled and phospho-disabled protein interactions. Because of the lack of T. gondii proteomes in Mechismo, the yeast and human orthologs of the phosphorylated proteins identified in our analysis were retrieved using the eggNOG-mapper ([PMC4702882] http://eggnogdb.embl.de/\#/app/emapper). In brief, relevant Orthologous Groups (OG) were firstly extracted and the yeast and human orthologs among these groups were finded out utilizing BioPython tools (http://biopython.org/). We then applied Clustal Omega [19] to align each orthologous sequence to the corresponding $T$. gondii protein and characterized the equivalent phosphorylation sites in the human or yeast proteins. Finally, they were input to Mechismo (http://mechismo.russelllab.org/) to characterize orthologous that enabled or disabled interactions in the human and yeast network.

\section{Results}

\section{Phosphorylation Profiles of T. gondii Oocysts}

In an effort to reveal genotype-specific differences in the phosphoproteomes of sporulated oocysts of $T$. gondii, we performed a comparative phosphoproteomic profiling of sporulated oocysts between virulent PYS strain and avriluent PRU strain. In total, we identified 10,645 unique phosphopeptides, 8,181 nonredundant phosphorylation sites and 2,792 phosphoproteins from sporulated oocysts of $T$. gondii virulent PYS strain and avriluent PRU strain with a FDR (false-discovery rate) $<0.01$ for phosphopeptide and phosphoRS probability $>0.75$ for phosphorylation site. The 8,181 phosphorylation sites consisted of 7,051 phosphoserine (86.27\%), 1,092 phosphothreonine (9.28\%), and 38 phosphotyrosine $(0.42 \%)$ (Fig. 1a). Out of the 10,645 phosphopeptides, 10,206 (95.88\%) phosphopeptides had one phosphorylation site, 426 phosphopeptides (4.00\%) had double phosphorylation sites, 12 phosphopeptide $(0.11 \%)$ possessed 3 triple phosphorylation sites and 1 phosphopeptide $(0.01 \%)$ possessed quadruple phosphorylation sites (Fig. 1b). The number of phosphopeptides matched with 1 spectrum, 2 spectra, 3 spectra, 4 spectra, 5 spectra, and $\geq 6$ spectra were 6, 201, 2, 124, 897, 462, 304, and 657, respectively (Fig. 1c). Additionally, the number of phosphoproteins harboring 1, 2, 3, 4, 5 and $\geq 6$ phosphorylation sites were 1,031, 550, 323, 208, 124 and 367, respectively (Fig. 1d). Among the total phosphoproteins, $99 \%$ phosphoproteins had CV (Coefficient of variation) value $<50 \%$ among three replicates (Fig. 2).

\section{Phosphopeptides Quantification and Clustering Analysis}


The quantitative analysis identified 4,129 differentially expressed phosphopeptides (DEPs) between sporulated oocysts of PYS strain and PRU strain (|log1.5 fold change| $>1$ and $p<0.05$ ), including 2,485 upregulated and 1,644 downregulated phosphopeptides (Fig. 3a, Additional file 1: Table S1 and Additional file 2: Table S2). Among these DEPs, 1,541, 665, 205, 61 and 13 phosphoproteins increased by $>1,2,3,4$, and $5 \log 1.5$ fold changes. In contrast, 1,273, 269, 82, 16 and 4 phosphoproteins decreased by $>1,2,3,4$ and $5 \log 1.5$ fold changes (Fig. 3b). The result of hierarchical clustering analysis of DEPs between sporulated oocysts of PYS strain and PRU strain is shown in Fig. 4.

\section{Motifs Analysis of Phosphorylation Sites of PYS Strain Versus PRU Strain}

Phosphosite motifs are crucial amino acid sequences, which are involved in the recognition of substrate by the corresponding kinase [16-17]. To investigate the potential motifs of upregulated or downregulated phosphorylated peptides of sporulated oocysts between PYS strain and PRU strain, substantial preference of amino-acid residue in sequence from -7 to +7 surrounding the phosphorylation sites was analyzed by the motif-X software tool. Motif analysis identified 24 motifs from the upregulated phosphorylated peptides in PYS strain when comparing PYS/PRU, including 22 serine motifs (VSP, SPR, SPxG, SPT, PxSP, LxxxRxxS, RxxSP, SDxE, SP, LxRxxS, RxxSxG, RxxSD, SExE, RxxS, SDxD, SGxE, GSE, DSD, GS, KxxS, SxxS and SxE) and 2 threonine motifs (TPE and TP) (Fig. 5). In regard to motif analysis of downregulated phosphorylated peptides in PYS strain when comparing PYS/PRU, 12 serine motifs (SPxG, AxSP, LSP, ExxxxxSP, DxSP, SPG, SPS, SPV, SP, RxxSL, RxxS and SxxxxxK) and 3 threonine motifs (TP, RxxT and KxxT) were overrepresented (Fig. 6). Because each motif corresponding to one or several category kinases, kinases consistent with motifs of upregulated or downregulated phosphorylated peptides are listed in Table 1 and Table 2, respectively. These results reflect the difference in the substrate-recognition abilities and kinase secretory preference of sporulated oocysts between virulent PYS strain and avirulent PRU strain. 
Table 1

Types of kinases represented by the motifs of up-regulated phosphor-peptides as determined by KinasePho.

\section{Motif Kinase Classes}

VSP PKA, PKB, PKG, CKI, IKK, CaM-II, ATM, MAPK, CDK, CDC2

SPR PKA, PKB, PKG, CKI, CKII, IKK, ATM, MAPK, CDK, CDC2, Syk, Jak, INSR

SP.G PKA, PKB, PKG, CKI, CKII, IKK, CaM-II, ATM, MAPK, CDK, CDC2, INSR

SPT PKA, PKB, PKG, CKI, IKK, ATM, MAPK, CDK, CDC2

P.SP PKA, PKB, PKG, CKI, IKK, ATM, MAPK, CDK, CDC2

L...R..S PKA, PKB, PKC, PKG, IKK, MAPK, CKI, CKII, CaM-II, ATM, CDC2

R..SP PKA, PKB, PKC, PKG, CDK, CDC2, MAPK, ATM, IKK, CKI, CKII, CaM-II, INSR

SD..E PKA, PKB, PKC, PKG, CKI, CKII, ATM, MAPK, IKK, CaM-II, EGFR, Src, CDC2

SP PKA, PKB, PKC, PKG, CKI, CKII, ATM, MAPK, IKK, CaM-II, CDK, CDC2, Jak, INSR, Syk, Src, Ab1

L.R..S PKA, PKB, PKC, PKG, CKI, CKII, IKK, CaM-II, CDK, CDC2, ATM

R..S.G PKA, PKB, PKC, PKG, CKI, CKII, IKK, CaM-II, CDK, CDC2, ATM, MAPK, INSR, SrC

R..SD PKA, PKB, PKC, PKG, CKI, CKII, IKK, CaM-II, CDC2, ATM, MAPK, INSR, EGFR, Syk

SE.E PKA, PKB, PKC, PKG, CKI, CKII, IKK, CaM-II, CDK, CDC2, ATM, MAPK, EGFR, INSR, Syk

R..S PKA, PKB, PKC, PKG, CKI, CKII, IKK, CaM-II, CDK, CDC2, ATM, MAPK, INSR, Syk

SD.D PKA, PKG, CKI, CKII, IKK, CDC2, ATM, MAPK, INSR

SG.E PKA, PKB, PKC, PKG, CKI, CKII, IKK, CaM-II, CDK, CDC2, ATM, MAPK, INSR, Syk

GSE PKA, PKG, CKI, CKII, IKK, CaM-II, CDC2, ATM, MAPK, Syk

DSD PKA, PKC, PKG, CKI, CKII, IKK, ATM, MAPK

GS PKA, PKB, PKC, PKG, CKI, CKIII, IKK, CaM-II, CDK, CDC2, ATM, MAPK, INSR, Syk, Src, Ab1

K..S PKA, PKB, PKC, PKG, CKI, CKII, IKK, CaM-II, CDK, CDC2, ATM, MAPK, EGFR, INSR

S..S PKA, PKB, PKC, PKG, CKI, CKIII, IKK, CaM-II, CDK, CDC2, ATM, MAPK, INSR, Syk, Src, Ab1

S.E PKA, PKC, PKG, CKI, CKII, IKK, CDK, CDC2, ATM, MAPK, INSR, Syk, Other_MDD

Abbreviations:

PKA: cAMP-dependent Protein Kinase; PKB: Protein Kinase B; PKC: Protein Kinase C; PKG: cGMPdependent Protein Kinase; CKI: Casein Kinase I; CKII: Casein Kinase Il; IKK: IkappaB Kinase; CaM-II: Calmodulin-dependent Protein Kinase II; ATM: Ataxia Telangiectasia Mutated Kinase; MAPK: MitogenActivated Protein Kinase; CDC2: Cell division Cycle Protein Kinase p34; CDK: Cyclin-Dependent Kinase; INSR: Insulin Receptor; EGFR: Epithelial Growth Factor Receptor; Syk: Cytoplasmic Tyrosine Kinases; Jak: Janus kinase; Src: Sarcoma gene kinase; Ab1: Abelson kinase . 


\section{Motif Kinase Classes}

TPE PKA, PKB, PKC, PKG, CKI, CKII, IKK, CaM-II, CDK, CDC2, ATM, MAPK, INSR, Syk, Src, Ab1

TP $\quad$ PKA, PKB, PKC, PKG, CKII, IKK, CaM-II, CDK, CDC2, ATM, MAPK, INSR

\section{Abbreviations.}

PKA: cAMP-dependent Protein Kinase; PKB: Protein Kinase B; PKC: Protein Kinase C; PKG: cGMPdependent Protein Kinase; CKI: Casein Kinase I; CKII: Casein Kinase Il; IKK: IkappaB Kinase; CaM-II: Calmodulin-dependent Protein Kinase Il; ATM: Ataxia Telangiectasia Mutated Kinase; MAPK: MitogenActivated Protein Kinase; CDC2: Cell division Cycle Protein Kinase p34; CDK: Cyclin-Dependent Kinase; INSR: Insulin Receptor; EGFR: Epithelial Growth Factor Receptor; Syk: Cytoplasmic Tyrosine Kinases; Jak: Janus kinase; Src: Sarcoma gene kinase; Ab1: Abelson kinase . 
Table 2

Types of kinases represented by the motifs of down-regulated phosphor-peptides as determined by

KinasePho.

\begin{tabular}{|c|c|}
\hline Motif & Kinase Classes \\
\hline SP.G & $\mathrm{PKA} \square \mathrm{PKB} \square \mathrm{PKC} \square \mathrm{PKG} \square \mathrm{CKI} \square \mathrm{CKII} \square \mathrm{IKK} \square \mathrm{CDK} \square \mathrm{CDC} 2 \square \mathrm{ATM} \square \mathrm{MAPK} \square A b 1$ \\
\hline A.SP & PKA $\square$ PKB $\square$ PKC $\square$ PKG $\square C K I I \square I K K \square C a M-I I \square C D K \square C D C 2 \square A T M \square M A P K \square I N S R \square S y k \square S r c \square A b 1$ \\
\hline LSP & PKA $\square$ PKB $\square$ PKC $\square$ PKG $\square C K I \square C K I I I K K \square C a M-I I \square C D K \square C D C 2 \square A T M \square M A P K$ \\
\hline E.....SP & 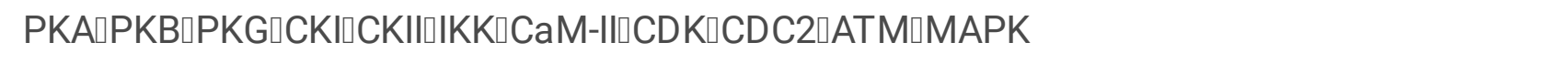 \\
\hline D.SP & PKA $\square$ PKG $\square K K \square C a M-I I \square C D C 2 \square A T M \square M A P K \square J a k$ \\
\hline SPG & $\mathrm{PKB} \square \mathrm{PKC} \square \mathrm{PKG} \square \mathrm{CKI} \square I \mathrm{KK} \square \mathrm{CaM}-I I \square \mathrm{CDK} \square \mathrm{CDC} 2 \square \mathrm{ATM} \square \mathrm{MAPK}$ \\
\hline SPS & PKA $\square$ PKB $\square$ PKC $\square$ PKG $\square C K I \square C K I I I K K \square C a M-I I C D K \square C D C 2 \square A T M \square M A P K \square I N S R \square S y k$ \\
\hline SPV & 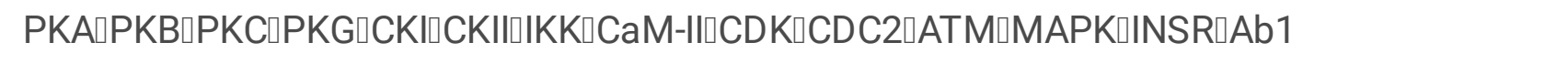 \\
\hline SP & PKA $\square$ PKB $\square$ PKC $\square$ PKG $\square C K I \square C K I I I K K \square C a M-I I \square C D K \square C D C 2 \square A T M \square M A P K \square E G F R \square I N S R \square S y k \square S r c$ \\
\hline R..SL & PKA $\square$ PKB $\square$ PKC $\square$ PKG $\square C K I \square I K K \square C a M-I I \square C D K \square C D C 2 \square A T M \square M A P K$ \\
\hline R..S & PKA $\square$ PKB $\square$ PKC $\square$ PKG $\square C K I \square C K I I I K K \square C a M-I I \square C D K \square C D C 2 \square A T M \square M A P K \square E G F R \square I N S R \square J a k \square S y k \square S r c$ \\
\hline S.....K & $\mathrm{PKA} \square \mathrm{PKB} \square \mathrm{PKC} \square \mathrm{PKG} \square \mathrm{CKI} \square \mathrm{CK} \| \square \mid \mathrm{KKK} \square \mathrm{CaM}-I \square C D K \square C D C 2 \square A T M \square M A P K \square I N S R \square J a k \square S y k$ \\
\hline TP & $\mathrm{PKA} \square \mathrm{PKB} \square \mathrm{PKC} \square \mathrm{PKG} \square \mathrm{CKI} \square C K I I \square I K K \square C a M-I \| C D K \square C D C 2 \square A T M \square M A P K \square J a k$ \\
\hline R..T & PKA $\square$ PKB $\square$ PKC $\square$ PKG $\square I K K \square C a M-I I C D C 2 \square M A P K \square E G F R \square I N S R \square S r C \square A b 1$ \\
\hline K..T & PKA $\square K C \square P K G \square C K I I I K K \square C D K \square M A P K$ \\
\hline \multicolumn{2}{|c|}{ Abbreviations: } \\
\hline \multicolumn{2}{|r|}{$\begin{array}{l}\text { PKA: cAMP-dependent Protein Kinase; PKB: Protein Kinase B; PKC: Protein Kinase C; PKG: cGMP- } \\
\text { dependent Protein Kinase; CKI: Casein Kinase I; CKIII: Casein Kinase Il; IKK: IkappaB Kinase; CaM-II: } \\
\text { Calmodulin-dependent Protein Kinase Il; ATM: Ataxia Telangiectasia Mutated Kinase; MAPK: Mitogen- } \\
\text { Activated Protein Kinase; CDC2: Cell division Cycle Protein Kinase p34; CDK: Cyclin-Dependent Kinase; } \\
\text { INSR: Insulin Receptor; EGFR: Epithelial Growth Factor Receptor; Syk: Cytoplasmic Tyrosine Kinases; } \\
\text { Jak: Janus Kinase; Src: Sarcoma Gene Kinase; Ab1: Abelson Kinase. }\end{array}$} \\
\hline
\end{tabular}

\section{Functional Enrichment Analysis}

To uncover the differences in the functions of the differentially expressed phosphoproteins (DEPs) of sporulated oocysts between PYS strain and PRU strain, GO enrichment analysis was performed. The enriched $\mathrm{GO}$ terms stratified into three $\mathrm{GO}$ categories (BP, CC and MF) are listed for upregulated and downregulated phosphoproteins (Fig. 7a-7b). The top five significantly enriched GO terms under BP for upregulated DEPs included catalytic activity, binding, transporter activity, structural molecule activity and transcription. In contrast, catalytic activity, binding, transporter activity, structural molecule activity and 
signal transducer activity were the top five enriched GO terms under BP for downregulated phosphoproteins.

In regard to the $\mathrm{CC}$ category, the five enriched $\mathrm{GO}$ terms of upregulated phosphoproteins were cell part, cell, organelle, membrane and macromolecular complex, whereas cell part, cell, organelle, macromolecular complex and membrane were the top five significantly enriched GO terms for the downregulated phosphoproteins. Regarding the MF of upregulated phosphoproteins, metabolic process, cellular process, localization, biological regulation and regulation of biological process were the top five enriched GO terms. In contrast, the five mostly enriched GO terms for downregulated phosphoproteins included metabolic process, cellular process, biological regulation, localization and regulation of biological process.

The co-expression networks of GO terms belonging to the three functional categories were constructed using the R Igraph package in order to investigate the level of association among GO terms. The significantly enriched GO terms in the network of upregulated phosphoproteins in PYS when comparing PYS/PRU are shown in Additional file 3: Figure S1. In the MF category, six GO terms, including pseudouridine synthase activity, 2-alkenal reductase $[N A D(P)]$ activity, metal ion transmembrane transporter activity, calcium ion transmembrane transporter activity, calcium-transporting ATPase activity, and motor activity, were significantly enriched. Also, in the CC category, six GO terms, including extracellular region, protein complex, membrane part, membrane protein complex, coated membrane, and membrane coat, were significantly enriched.

In the BP category, $40 \mathrm{GO}$ terms were significantly enriched, including biological regulation, regulation of biological process, cell cycle process, response to stress, transmembrane transport, G-protein coupled receptor signaling pathway, glycerolipid metabolic process, glycerophospholipid metabolic process, phosphatidylinositol metabolic process, lipid phosphorylation, phosphatidylinositol phosphorylation, organophosphate metabolic process, aromatic compound catabolic process, cellular nitrogen compound catabolic process, heterocycle catabolic process, nucleobase-containing compound catabolic process, organic cyclic compound catabolic process, negative regulation of biological process, negative regulation of cellular process, regulation of cellular component organization, regulation of organelle organization, RNA modification, pseudouridine synthesis, regulation of metabolic process, regulation of cellular metabolic process, regulation of primary metabolic process, regulation of biosynthetic process, regulation of nitrogen compound metabolic process, regulation of cellular biosynthetic process, regulation of cellular amide metabolic process, regulation of macromolecule metabolic process, regulation of RNA metabolic process, regulation of macromolecule biosynthetic process, regulation of RNA biosynthetic process, regulation of cellular macromolecule biosynthetic process, regulation of nucleic acid-templated transcription, regulation of translation, regulation of gene expression, regulation of transcription, and posttranscriptional regulation of gene expression.

The significantly enriched GO terms in the network of downregulated phosphoproteins in PYS when comparing PYS/PRU are shown in Additional file 4: Figure S2. In the MF category, $17 \mathrm{GO}$ terms, including 
binding, small molecular binding, nucleoside phosphate binding, nucleotide binding, pattern binding, polysaccharide binding, starch binding ligase activity, transferase activity, oxidoredutase, kinase regulatory activity, protein kinase regulatory activity, antiporter activity, cation antiporter activity, proton antiporter activity, translation initiation factor activity and translation factor activity, were significantly enriched. Only three GO terms (cell, cell part and intracellular) in the CC category were enriched. However, in the BP category there were 21 significantly enriched GO terms, including locomotion, developmental process, cellular developmental process, anatomical structure morphogenesis, anatomical structure development, tissue development, nervous system development, response to external stimulus, regulation of phosphorus metabolic process, regulation of phosphate metabolic process, regulation of phosphorylation, regulation of protein phosphorylation, regulation of protein kinase activity, regulation of kinase activity, macromolecule metabolic process, pyruvate metabolic process, cellular carbohydrate biosynthetic process, cellular amide metabolic process, peptide metabolic process, peptide biosynthetic process, and translation metabolic process establishment of localization and transport.

Compared to PRU strain, more upregulated phosphoproteins were enriched in $\mathrm{GO}$ terms such as transcription regulator activity, macromolecular complex, immune system process, biological adhesion in PYS strain. However, more downregulated phosphoproteins were enriched in $\mathrm{GO}$ terms such as signal transducer activity, developmental process, reproductive process, reproduction, locomotion and pigmentation. These results clearly show significant differences in the biological functions of the DEPs of sporulated oocysts between PYS strain and PRU strain.

\section{KEGG Pathway Analysis}

Enzymes with phosphorylation sites can regulate key signal pathways in several organisms [11]. To better understand the signal pathways controlled by the phosphorylated proteins and the function of the DEPs, DEPs in sporulated oocysts between PYS strain and PRU strain were annotated and mapped against in the KEGG pathway database. As shown in Figs. 8, 39 and 18 DEPs were significantly enriched in RNA transport and phosphatidylinositol signaling system, respectively.

\section{Protein-Protein Interaction (PPI) Analysis}

Using Cytoscape software, the PPI networks (combined score $\geq 0.9$ ) were built in order to identify the mechanisms regulated by phosphorylation and to determine the related functional clusters of the DEPs of sporulated oocysts between the virulent and avirulent $T$. gondii strains. The PPI network of upregulated and downregulated phosphoproteins between PRU strain and PYS strain included 344 nodes and 670 interactor edges (Fig. 9). Several major hubs were identified, including ribosome biogenesis protein BOP1 (TGME49_301390), nucleolar protein NOP5 (TGME49_205510), eukaryotic initiation factor-2

(TGME49_313230), translation initiation factor 2 beta (TGME49_235540), eukaryotic translation initiation factor 2 gamma subunit (TGME49_235970), translation elongation factor 2 family protein (TGME49_205470), ribosomal protein RPL6 (TGME49_313390), ribosomal protein RPL4 (TGME49_309120), and ATP-dependent RNA helicase (TGME49_312280). Several functional clusters were identified in the PPI network, including DNA replication or RNA transcription, proteasome, 
metabolism, RNA splicing or translation, ribosomal proteins, molecular chaperones, tRNA related molecular, adapter, coatomer and kinases or enzymes.

\section{Kinase Related Networks}

Given that phosphorylation influences the activity of the phosphorylated protein that function as a kinase, potential peptides whose phosphorylation is related to the respective kinase were identified by performing a correlation analysis (Additional file 5: Table S3). After retaining phosphopeptides with $>\sim 1.5$-fold change abundance, 2 phosphorylated kinases were identified and were associated with 58 phosphopeptides. Positive correlation and negative correlation indicate that kinase phosphorylation positively or negatively impacts its action. These correlations suggest potential differences in kinases and associated substrates of sporulated oocysts between the two T. gondii strains. Among the first cluster of phosphorylated peptides, peptides related to T. gondii AGC kinase A0A125YVN2 (connected with 50 phosphorylated peptides) connected most kinase peptides. The second cluster of phosphorylated peptides encompassed 6 proteins: zinc finger A0A125YGX1, TgCatPRC2 A0A151H897, possible RNAbinding protein Q1 JTD1, DNA-directed RNA polymerase subunit S8F5G5, non-specific serine/threonine protein kinase S8F5G5 and the hub protein kinase is histone kinase SNF1 S8F5G5 (Fig. 10). The GO annotation of the most connected kinases peptides (AGC kinase) showed GO terms associated with nucleotide binding, ATP binding, metabolic process, protein serine/threonine kinase activity and phosphorylation, in the MF category.

\section{Potential Phosphorylation-Dependent Interactions}

Based on the T. gondii protein interaction network, the putative function of the different phosphorylation sites in our dataset were sought to identify and the effects of mutation or phosphorylation on the interaction between two phosphorylated proteins (enable or disable) were predicted. Using Mechismo and Pearson correlational analysis, a total of 147 interactors of the $T$. gondii orthologs, enabled or disabled by phosphorylation of the identified sites, were identified (Additional file 6: Table S4). The 9 interacting partners were annotated back to $T$. gondii proteins and were found to influence interactors in $T$. gondii (Fig. 10). The T6 and T12 phosphosites of T. gondii A0A151 H503 gene were found to influence 7 interactions and phosphosite S10 of T. gondii A0A151GZW7 gene influenced 2 interactions.

\section{Discussion}

In this study, we used iBT-based mass-spectrometry approach combined with $\mathrm{TiO}_{2}$ affinity chromatography to profile the phosphoproteomic landscape of sporulated oocysts of virulent (Chinese ToxoDB\#9, PYS strain) and avirulent strains (type II, PRU strain) of T. gondii. Our results are consistent with previous phosphoproteomic studies in eukaryotic organisms [22-24]. We identified 10,645 phosphopeptides, 2,792 phosphoproteins and 8,181 phosphorylation sites, including 9,574 (86.27\%) phosphoserine (pSer), 1,477 (9.28\%) phosphothreonine (pThr), and $47(0.42 \%)$ phosphotyrosine (pTyr) in the sporulated oocysts of T. gondii virulent PYS strain and avriluent PRU strain (Fig. 1). This finding is 
anticipated because most $T$. gondii proteins containing serine kinase domains, such as ROP5, ROP16, ROP18 and TgSR3, play roles in the replication, invasion and virulence of this parasite [25-27]. Threonine phosphorylation was associated with serine phosphorylation in several virulence factors of $T$. gondii, such as ROP18 and type $2 \mathrm{C}$ serine-threonine phosphatase and can play role in the invasion and replication of $T$. gondii [28-29]. Although tyrosine phosphorylation was underrepresented compared to serine and threonine phosphorylation in the apicomplexan parasites, it contributes to important signaling pathways, such as the MAPK pathway $[13,30]$.

\section{Motifs}

Motif analysis identified 24 motifs for the upregulated phosphorylated peptides, and 15 motifs for the downregulated phosphorylated peptides in PYS strain compared to PRU strain (Fig. 5 and Fig. 6). The motif's function is to recognize the corresponding substrate by kinase, and every motif corresponds to one or several enzymes [20-21,31]. Therefore, different motifs extracted from upregulated and downregulated phosphorylated peptides not only show the differences in the kinase expression of sporulated oocysts between PYS stain and PRU strain, but also reflect the discrepancy in the substraterecognition abilities of sporulated oocysts between PYS and PRU strains.

Our results showed that these motifs are consistent with some traditional kinases (PKA; PKG; CK II; IKK; CDC2; MAPK), which play roles in the egress, motility, cell cycle, and stress response of T. gondii [32-35]. Some less prevalent kinases were also consistent with these motifs, such as EGFR, INSR, Jak, Syk, Src, Ab1. The protein kinase epidermal growth factor receptor (EGFR) is found on cell surface in the Apicomplexan parasite Neospora caninum. The $N$. caninum EGFR can be activated by NcMIC3 which also contains four EGF domains, or human EGF. Interestingly, the activation of EGFR in N. caninum enhanced the proliferation of tachyzoites [36]. INSR can participate in the insulin-like growth factor signaling pathway and was identified as a good candidate for parasitic RTK inhibition [37-39].

Syk kinases, identified in invertebrates, regulate the proliferation, differentiation, and survival of various types of cells. Among Syk kinase family, tyrosine kinase 4 (TK4) is a kinase expressed in Schistosoma mansoni and $S$. japonicum, which plays a pivotal role in the development of the testis and ovaries in $S$. mansoni and regulates the gametogenesis of S. japonicum [40]. The fact that Src inhibitors can affect the actin cytoskeleton of Entamoeba histolytica and inhibit the entry of Trypanosoma cruzi provides new targets for designing new drugs against protozoa [41-42]. The Abl family kinases not only can play a role in the signaling translation from growth factor and adhesion receptors into cytoskeletal rearrangements, but also can facilitate some processes, such as endocytosis, viral and bacterial uptake, autophagy, and IgG-mediated phagocytosis. Also, this kinase was shown to facilitate Leishmania amazonensis amastigote uptake by macrophages [43]. The important role of these kinases in parasites implies the potential role of phosphorylated protein in the sporulated oocyst of $T$. gondii, which warrants further investigation.

\section{GO and KEGG analysis of the phosphorylated proteins}


GO analysis revealed that transcription regulatory activity, macromolecular complex, immune system process, biological adhesion $\mathrm{GO}$ terms were enriched in more upregulated phosphoproteins in PYS strain when comparing PYS/PRU. However, the GO terms signal transducer activity, developmental process, reproductive process, reproduction, locomotion and pigmentation were more enriched in downregulated phosphoproteins. These results show the difference in the transcription regulation and developmental regulation of sporulated oocysts between PYS strain and PRU strain. Phosphorylation of translational regulators was associated with the reprogramming of the transcriptome of higher organisms [44]. Our results indicate that this post-translational protein modification exists in sporulated oocysts of $T$. gondii. The glideosome-associated protein with multiple-membrane spans (GAPM3) is a part of glideosome, a macromolecular complex consisting of adhesive proteins [45], which was an upregulated DEP in PYS strain when comparing PYS/PRU in our data. Substrate-dependent locomotion known as gliding motility is a unique form which is essential for invading host cells and traversing the environment of $T$. gondii [46]. Several Toxoplasma gondii MyoA motility complex were found to be phosphorylated in our results, for example myosin $\mathrm{A}$, myosin $\mathrm{C}$, myosin $\mathrm{F}$, myosin $\mathrm{G}$ and myosin $\mathrm{J}$. Of which, phosphorylated peptide of myosin $\mathrm{F}$ was downregulated in PYS stain when comparing PYS/PRU. As for the results of KEGG pathway analysis, phosphatidylinositol signaling system is a significantly enriched pathway. This pathway was deemed as crucial constitutive signals that regulate biochemical organelle identity at eukaryotic cellular membranes, as well as autophagy and endocytosis in protist parasites such as $T$. gondii [47-48].

\section{Protein-Protein Interaction (PPI) Networks}

Among the functional clusters identified in the PPI network, some well-known clusters (e.g. DNA replication or RNA transcription, metabolism, RNA splicing or translation, ribosomal proteins, molecular chaperones, tRNA related molecular, and kinases or enzymes) consist of phosphorylated proteins not only were identified, but also the unusual clusters, such as proteasome, adapter, coatomer, were included. The main function of proteasome is proteolytic removal of damaged proteins and this large intracellular protein complex has a crucial role in the pathogenesis, intracellular growth and replication of $T$. gondii [49-50]. The presence of proteasome cluster in the PPI network demonstrates that phosphorylation of proteins in the proteasome might contribute to the pathogenesis, intracellular growth and replication of $T$. gondii. The adaptor complex of all eukaryotes is composed of receptor/adaptor proteins that mediate the recruitment of dynamin-related protein, the key player of mitochondrial fission, to the mitochondrial outer membrane (MOM) at ER/actin preconstricted fission sites [51]. So, this complex is critical for the reconstitution of the mitochondria membrane. The endodyogeny of tachyzoites of $T$. gondii is tightly linked with duplication of the mitochondrion [51]. Although there was no evidence that the division of sporulated oocysts of $T$. gondii was associated with phosphorylation of adaptor proteins, the essential of phosphorylation of adaptor proteins for its function is an interesting event to be survey. Coatomer coated complex (COP) is a type of transport vesicles, which mediates both selective and non-selective transport within the eukaryotic cell including the eukaryotic protozoan $T$. gondii. In fact, $T$. gondii has seven subunit homologues and the size of these homologues were different [52-53]. Our analysis showed that four subunits of COP were phosphorylated, including alpha, beta, gamma and zeta. This high percentage of 
phosphorylation in the subunits of COP indicates that phosphorylation may be necessary for the function of COP and transport of vesicles of T. gondii.

\section{Kinase-Protein Interactions}

Kinase phosphorylate substrates in order to modulate activity and interactions of proteins. In our study, specific parasite peptides associated with the phosphorylated peptides were identified and the potential kinase-protein interactions were predicted. The parasite kinase (AGC kinase) had the greatest connected peptides. AGC kinase represents a category conserved kinases among eukaryotic genomes, including cyclic AMP [cAMP]-dependent protein kinase 1 (protein kinase A), cGMP-dependent protein kinase (PKG) and protein kinase $\mathrm{C}$, which served various physiological functions, such as cell growth, metabolism, differentiation, and cell death [54]. This kinase was widely expressed in Apicomplexan parasites and contribute to the pathogenesis of parasites [55]. Our data revealed that AGC kinase of sporulated oocyst of $T$. gondii had regulatory roles in nucleotide binding, ATP binding, metabolic process, protein serine/threonine kinase activity and phosphorylation, indicating the important role of AGC kinase in the biology of T. gondii sporulated oocysts.

\section{Conclusions}

The present study revealed the global phosphoproteomic differences in sporulated oocysts between virulent and avirulent $T$. gondii strains of different genotypes using the iBT technology combined with $\mathrm{TiO}_{2}$ affinity chromatography. Abundant phosphopeptides in sporulated oocysts between virulent and avirulent $T$. gondii strains exhibited distinct difference in the conserved motifs, GO terms, enriched pathways and PPI networks. The kinase associated network analysis indicated the role of phosphorylation in the transformation of $T$. gondii kinases. AGC kinase was the most connected kinases peptides. These data provide new insight into the phenotypic differences in sporulated oocysts of virulent and avirulent $T$. gondii strains.

\section{Abbreviations}

Ab1: Abelson kinase; ACN: acetonitrile; AGC: automatic gain control; ATM: Ataxia Telangiectasia Mutated Kinase; BOP: ribosome biogenesis protein; CaM-II: Calmodulin-dependent Protein Kinase II; CDC2: Cell division Cycle Protein Kinase p34; CDK: Cyclin-Dependent Kinase; CKI: Casein Kinase I; CKII: Casein Kinase II; COP: coatomer coated complex; CsCl: caesium chloride; CV: coefficient of variation; DEPs: differentially expressed phosphopeptides; DTT: dithiothreitol; EGFR: Epithelial Growth Factor Receptor; FA: formic acid; FDR: false-discovery rate; GO: Gene Ontology; HPLC : high-performance liquid chromatography; IAM: iodoacetamide; iBT: isobaric tag; IKK: IkappaB Kinase; INSR: Insulin Receptor; iTRAQ: isobaric tag for relative and absolute quantitation; Jak: Janus kinase; KEGG: Kyoto Encyclopedia of Genes and Genomes; LC-MS: liquid chromatography-mass spectrometry; MAPK: Mitogen-Activated Protein Kinase; MAT: modified agglutination test; MOM: mitochondrial outer membrane; MS/MS: tandem mass spectrometry; NOP: nucleolar protein; OG: Orthologous Groups; PBS: phosphate-buffered saline; 
PKA: cAMP-dependent Protein Kinase; PKB: Protein Kinase B; PKC: Protein Kinase C; PKG: cGMPdependent Protein Kinase; PPI: protein-protein interaction; PTM: post-translational modification; ROP: rhoptry protein; SPF: specific-pathogen-free; Src: Sarcoma gene kinase; STRING: Search Tool for the Retrieval of Interacting Genes/Protein; TFA: trifluoroacetic acid; $\mathrm{TiO}_{2}:$ Titanium dioxide; TK: tyrosine kinase; Syk: Cytoplasmic Tyrosine Kinases.

\section{Declarations}

\section{Acknowledgments}

We thank BGI-Shenzhen for technical assistance with the LC-MS/MS analysis.

\section{Ethical approval and consent to participate}

All animal experiments were strictly performed according to the instructions set forth in the Animal Ethics Procedures and Guidelines of the People's Republic of China. Animals were obtained from the Laboratory Animal Center of Lanzhou Veterinary Research Institute (LVRI). All animal experiments were inspected and granted by the Animal Administration and Ethics Committee of LVRI, Chinese Academy of Agricultural Sciences. Earnest endeavors was conducted to reduce animal affliction and to minimize the quantity of animals used in the experiment.

\section{Consent for publication}

Not applicable.

\section{Availability of data and materials}

The datasets supporting the findings of this article are included within the article. All the mass spectrometry data have been deposited to the ProteomeXchange Consortium with identifier PXD017032.

\section{Competing interests}

The authors declare that they have no competing interests.

\section{Funding}

Project financial support was kindly provided by the Open Funds of the State Key Laboratory of Veterinary Etiological Biology, Lanzhou Veterinary Research Institute, Chinese Academy of Agricultural Sciences (Grant No. SKLVEB2019KFKT005), the International Science and Technology Cooperation Project of Gansu Province (Grant Nos. 17JR7WA031), and the Agricultural Science and Technology Innovation Program (ASTIP) (Grant No. CAAS-ASTIP-2016-LVRI-03).

\section{Authors' contributions}


ZXW, HME, XQZ and XLS conceived and designed the research. ZXW performed the experiments, analyzed the data and wrote the paper. RSH and XLS contributed reagents/materials/analysis tools. ZXW, HME and XQZ critically revised the manuscript. All authors read and approved the final version of the manuscript.

\section{Author details}

${ }^{1}$ College of Veterinary Medicine, Gansu Agricultural University, Lanzhou, Gansu Province 730070, PR China. ${ }^{2}$ State Key Laboratory of Veterinary Etiological Biology, Key Laboratory of Veterinary Parasitology of Gansu Province, Lanzhou Veterinary Research Institute, Chinese Academy of Agricultural Sciences, Lanzhou, Gansu Province 730046, PR China.

${ }^{3}$ College of Animal Science and Technology, Jilin Agricultural University, Changchun, Jilin Province 130118, PR China. ${ }^{4}$ Jiangsu Co-innovation Center for the Prevention and Control of Important Animal Infectious Diseases and Zoonoses, Yangzhou University College of Veterinary Medicine, Yangzhou, Jiangsu Province 225009, PR China. ${ }^{5}$ Faculty of Medicine and Health Sciences, School of Veterinary Medicine and Science, University of Nottingham, Sutton Bonington Campus, Loughborough, LE12 5RD, UK.

\section{References}

1. Wang ZT, Verma SK, Dubey JP, Sibley LD. The aromatic amino acid hydroxylase genes AAH1 and AAH2 in Toxoplasma gondii contribute to transmission in the cat. PLoS Pathog. 2017; 13: e1006272.

2. Behnke MS, Zhang TP, Dubey JP, Sibley LD. Toxoplasma gondii merozoite gene expression analysis with comparison to the life cycle discloses a unique expression state during enteric development. BMC Genomics. 2014; 15: 350.

3. Herrmann DC, Bärwald A, Maksimov A, Pantchev N, Vrhovec MG, Conraths FJ, et al. Toxoplasma gondii sexual cross in a single naturally infected feline host: generation of highly mouse-virulent and avirulent clones, genotypically different from clonal types I, II and III. Vet Res. 2012; 43: 39.

4. Wang ZX, Zhou CX, Elsheikha HM, He S, Zhou DH, Zhu XQ. Proteomic differences between developmental stages of Toxoplasma gondii revealed by iTRAQ-based quantitative proteomics. Front Microbiol. 2017; 8: 985.

5. Saeij JP, Boyle JP, Boothroyd JC. Differences among the three major strains of Toxoplasma gondii and their specific interactions with the infected host. Trends Parasitol. 2005; 21: 476-481.

6. Sibley LD, Boothroyd JC. Virulent strains of Toxoplasma gondii comprise a single clonal lineage. Nature. 1992; 359: 82-85.

7. Cheng WS, Liu F, Li M, Hu XD, Chen H, Pappoe F, et al. Variation detection based on next-generation sequencing of type Chinese 1 strains of Toxoplasma gondii with different virulence from China. BMC Genomics. 2015; 16: 888. 
8. Lorenzi H, Khan A, Behnke MS, Namasivayam S, Swapna LS, Hadjithomas M, et al. Local admixture of amplified and diversified secreted pathogenesis determinants shapes mosaic Toxoplasma gondii genomes. Nat. Commun. 2016; 7: 10147.

9. Zhang Y, Jiang N, Zhang T, Wang D, Feng Y, Sang X, et al. Toxoplasma gondii genotype determines Tim-3 expression levels in splenic and circulatory T cells in mice. Front. Microbiol. 2018; 9: 2967.

10. 10. Zhou DH, Wang ZX, Zhou CX, He S, Elsheikha HM, Zhu XQ. Comparative proteomic analysis of virulent and avirulent strains of Toxoplasma gondii reveals strain-specific patterns. Oncotarget. 2017; 8: 80481-80491.

11. Wang ZX, Zhou CX, Calderón-Mantilla G, Petsalaki E, He JJ, Song HY, et al. iTRAQ-based global phosphoproteomics reveals novel molecular differences between Toxoplasma gondii strains of different genotypes. Front. Cell. Infect Microbiol. 2019; 9: 307.

12. Ren S, Yang M, Li Y, Zhang F, Chen Z, Zhang J, et al. Global phosphoproteomic analysis reveals the involvement of phosphorylation in aflatoxins biosynthesis in the pathogenic fungus Aspergillus flavus. Sci Rep. 2016; 6: 34078.

13. Treeck M, Sanders JL, Elias JE, Boothroyd JC. The phosphoproteomes of Plasmodium falciparum and Toxoplasma gondii reveal unusual adaptations within and beyond the parasites' boundaries. Cell Host Microbe. 2011; 10: 410-419.

14. 14. Staggs SE, See MJ, Dubey JP, Villegas EN. Obtaining highly purified Toxoplasma gondii oocysts by a discontinuous cesium chloride gradient. J Vis Exp. 2009; 3:

15. Dumètre A, Dardé ML. Purification of Toxoplasma gondii oocysts by cesium chloride gradient. J Microbiol Methods. 2004; 56: 427-430.

16. Zhou CX, Zhu XQ, Elsheikha HM, He S, Li Q, Zhou DH, et al. Global iTRAQ-based proteomic profiling of Toxoplasma gondii oocysts during sporulation. J. Proteomics. 2016; 148: 12-19.

17. 17. Finn RD, Coggill P, Eberhardt RY, Eddy SR, Mistry J, Mitchell AL, et al. The Pfam protein families database: towards a more sustainable future. Nucleic Acids Res. 2016; 44(D1): D279-2

18. Betts MJ, Lu Q, Jiang Y, Drusko A, Wichmann O, Utz M, et al. Mechismo: predicting the mechanistic impact of mutations and modifications on molecular interactions. Nucleic Acids Res. 2015; 43: e10.

19. Sievers F, Wilm A, Dineen D, Gibson TJ, Karplus K, Li W, et al. Fast, scalable generation of high-quality protein multiple sequence alignments using Clustal Omega. Mol Syst Biol. 2011; 7: 539.

20. Schwartz D, Gygi SP. An iterative statistical approach to the identification of protein phosphorylation motifs from large-scale datasets. Nat. Biotechnol. 2005; 23: 1391-1398.

21. Pi E, Qu L, Hu J, Huang Y, Qiu L, Lu H, et al. Mechanisms of soybean roots' tolerances to salinity revealed by proteomic and phosphoproteomic comparisons between two cultivars. Mol Cell Proteomics. 2016; 15: 266-288.

22. Pearlman SM, Serber Z, Ferrell JEJr. A mechanism for the evolution of phosphorylation sites. Cell. 2011; 147: 934-946. 
23. 23. Huttlin EL, Jedrychowski MP, Elias JE, Goswami T, Rad R, Beausoleil SA, et al. A tissue-specific atlas of mouse protein phosphorylation and expression. Cell. 2010; 143: 1174-11

24. Olsen JV, Blagoev B, Gnad F, Macek B, Kumar C, Mortensen P, et al. Global, in vivo, and site-specific phosphorylation dynamics in signaling networks. Cell. 2006; 127: 635-648.

25. Behnke MS,Khan A,Wootton JC, Dubey JP, Tang K, Sibley LD. Virulence differences in Toxoplasma mediated by amplification of a family of polymorphic pseudokinases. Proc Natl Acad Sci USA. 2011; 108: 9631-9636.

26. Taylor S,Barragan A,Su C, Fux B, Fentress SJ, Tang K, et al. A secreted serine- threonine kinase determines virulence in the eukaryotic pathogen Toxoplasma gondii. Science.2006; 314: 1776-1780.

27. Yeoh LM, Goodman CD, Hall NE, van Dooren GG, McFadden GI, Ralph SA. A serine-arginine-rich (SR) splicing factor modulates alternative splicing of over a thousand genes in Toxoplasma gondii. Nucleic Acids Res. 2005; 43: 4661-4675.

28. Du J, An R, Chen L, Shen Y, Chen Y, Cheng L, et al. Toxoplasma gondii virulence factor ROP18 inhibits the host NF-KB pathway by promoting p65 degradation. J Biol Chem. 2014; 289: 12578-12592.

29. Jan G, Delorme V, Saksouk N, Abrivard M, Gonzalez V, Cayla X, et al. A Toxoplasma type $2 \mathrm{C}$ serinethreonine phosphatase is involved in parasite growth in the mammalian host cell. Microbes Infect. 2009; 11: 935-945.

30. Lim WA, Pawson T. Phosphotyrosine signaling: evolving a new cellular communication system. Cell. 2010; 142: 661-667.

31. Nakayasu ES, Tempel R, Cambronne XA, Petyuk VA, Jones MB, Gritsenko MA, et al. Comparative phosphoproteomics reveals components of host cell invasion and post-transcriptional regulation during Francisella infection. Mol Cell Proteomics. 2013; 12: 3297-3309.

32. 32. Delorme V, Cayla X, Faure G, Garcia A, Tardieux I. Actin dynamics is controlled by a casein kinase II and phosphatase 2C interplay on Toxoplasma gondii Mol Biol Cell. 2003; 14: 1900-1912.

33. 33. Wei F, Wang W, Liu Q. Protein kinases of Toxoplasma gondii: functions and drug targets. Parasitol Res. 2013; 112: 2121-2129.

34. Molestina RE, Sinai AP. Detection of a novel parasite kinase activity at the Toxoplasma gondii parasitophorous vacuole membrane capable of phosphorylating host IkappaBalpha. Cell Microbiol. 2005; 7: 351-362.

35. Khan F, Tang J, Qin CL, Kim K. Cyclin-dependent kinase TPK2 is a critical cell cycle regulator in Toxoplasma gondii. Mol Microbiol. 2002; 45: 321-332.

36. Jin X, Li G, Zhang X, Gong P, Yu Y, Li J. Activation of a Neospora caninum EGFR-like kinase facilitates intracellular parasite proliferation. Front Microbiol. 2017; 8: 1980.

37. Gomes CM, Goto H, Magnanelli AC, Monteiro HP, Soares RP, Corbett CE, et al. Characterization of the receptor for insulin-like growth factor on Leishmania promastigotes. Exp Parasitol. 2001; 99: 190197. 
38. Li J, Xiang M, Zhang R, Xu B, Hu W. RNA interference in vivo in Schistosoma japonicum: Establishing and optimization of RNAi mediated suppression of gene expression by long dsRNA in the intramammalian life stages of worms. Biochem Biophys Res Commun. 2018; 503: 1004-1010.

39. Vicogne J, Dissous C. Schistosoma mansoni receptor tyrosine kinases: towards new therapeutic targets. J Soc Biol. 2003; 197: 367-373.

40. Ding H, Liu F, Zhu L, Wu F, Liu Q, He S, et al. Tyrosine kinase 4 is involved in the reproduction of the platyhelminth parasite Schistosoma japonicum. Parasit Vectors. 2017; 10: 498.

41. López-Contreras L, Hernández-Ramírez VI, Flores-García Y, Chávez-Munguía B, Talamás-Rohana P. Src and PI3 K inhibitors affect the virulence factors of Entamoeba histolytica. Parasitology. 2013; 140: 202-209.

42. Melo TG, Tucci AR, Nogueira AR, Meirelles MdeN, Pereira MC. The involvement of FAK and Src in the invasion of cardiomyocytes by Trypanosoma cruzi. Exp Parasitol. 2014; 139: $49-57$.

43. Wetzel DM, Rhodes EL, Li S, McMahon-Pratt D, Koleske AJ. The Src kinases Hck, Fgr and Lyn activate Arg to facilitate IgG-mediated phagocytosis and Leishmania infection. J Cell Sci. 2016; 129: 31303143.

44. Spoel SH, Tada Y, Loake GJ. Post-translational protein modification as a tool for transcription reprogramming. New Phytol. 2010; 186: 333-339.

45. Keeley A, Soldati D. The glideosome: a molecular machine powering motility and host-cell invasion by Apicomplexa. Trends Cell Biol. 2004; 14: 528-532.

46. Powell CJ, Jenkins ML, Parker ML, Ramaswamy R, Kelsen A, Warshaw DM, et al. Dissecting the molecular assembly of the Toxoplasma gondii MyoA motility complex. J Biol Chem. 2017; 292: 19469-19477.

47. Wengelnik K, Daher W, Lebrun M. Phosphoinositides and their functions in apicomplexan parasites. Int J Parasitol. 2018; 48: 493-504.

48. Cernikova L, Faso $C$, Hehl AB. Roles of phosphoinositides and their binding proteins in parasitic protozoa. Trends Parasitol. 2019; 35: 996-1008.

49. Shaw MK, He CY, Roos DS, Tilney LG. Proteasome inhibitors block intracellular growth and replication of Toxoplasma gondii. Parasitology. 2000; 121: 35-47.

50. Paugam A, Creuzet C, Dupouy-Camet J, Roisin MP. Evidence for the existence of a proteasome in Toxoplasma gondii: intracellular localization and specific peptidase activities. Parasite. 2001 ; 8: 267273.

51. Melatti C, Pieperhoff M, Lemgruber L, Pohl E, Sheiner L, Meissner M. A unique dynamin-related protein is essential for mitochondrial fission in Toxoplasma gondii. PLoS Pathog. 2019; 15: e1007512.

52. Pfluger SL, Goodson HV, Moran JM, Ruggiero CJ, Ye X, Emmons KM, et al. Receptor for retrograde transport in the apicomplexan parasite Toxoplasma gondii. Eukaryot Cell. 2005; 4: 432-442. 
53. Smith SS, Pfluger SL, Hjort E, McArthur AG, Hager KM. Molecular evolution of the vesicle coat component betaCOP in Toxoplasma gondii. Mol. Phylogenet Evol. 2007; 44: 1284-1294.

54. Wang Y, Deng YZ, Cui G, Huang C, Zhang B, Chang C, et al. The AGC kinase SsAgc1 regulates Sporisorium scitamineum mating/filamentation and pathogenicity. mSphere. 2019; 4: e00259-19.

55. Haidar M, Ramdani G, Kennedy, EJ, Langsley G. PKA and Apicomplexan parasite diseases. Horm Metab Res. 2017; 49: 296-300.

\section{Figures}

a

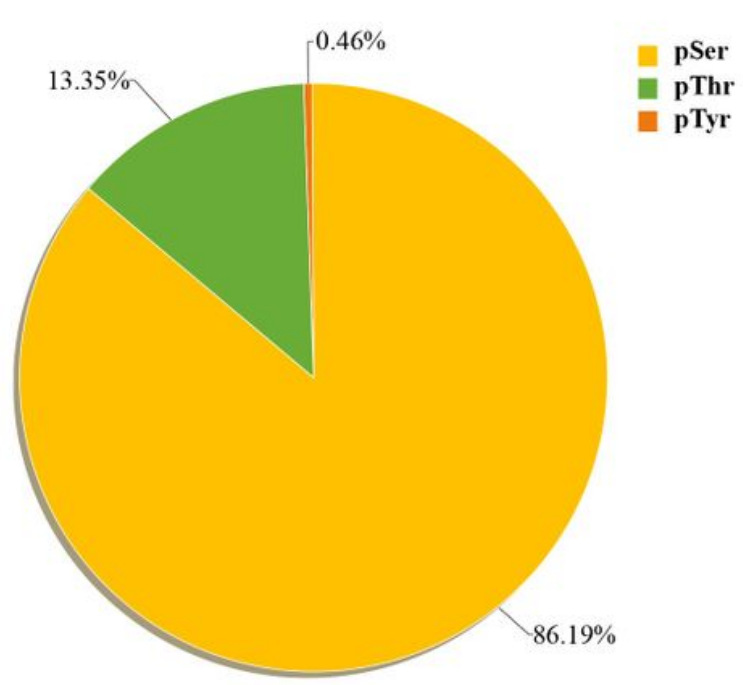

c

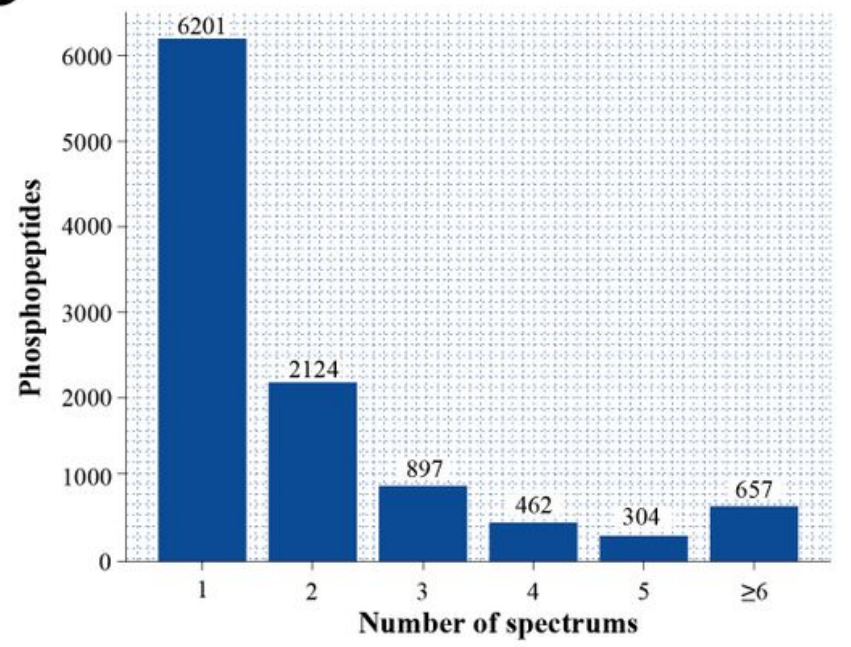

b

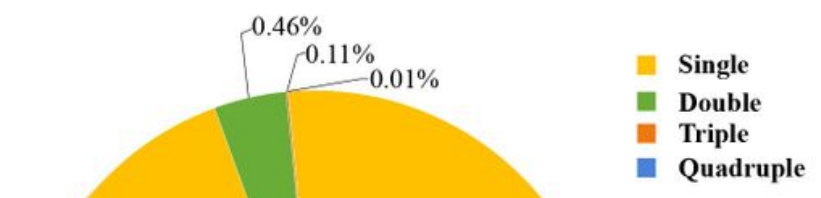

d

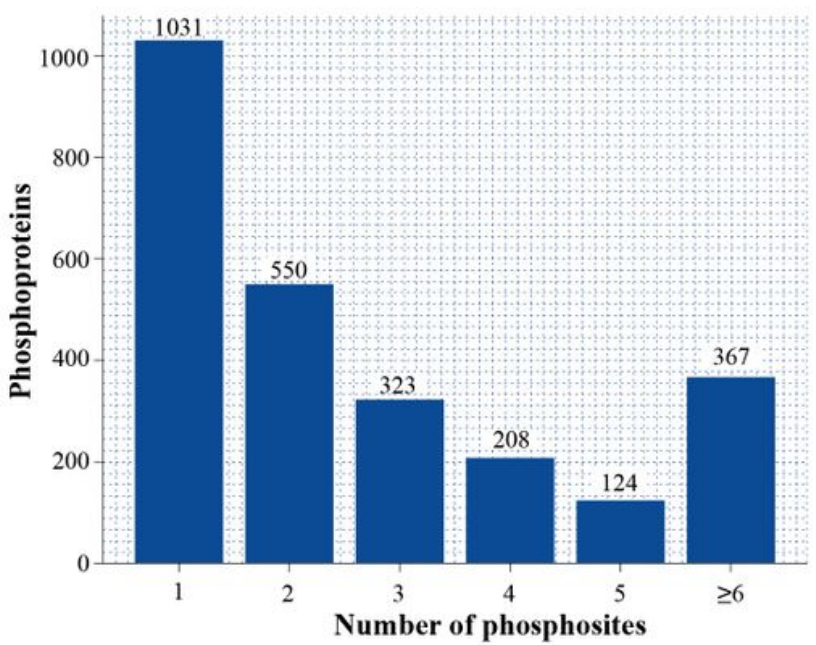

\section{Figure 1}

Summary of phosphoproteomic parameters of sporulated oocysts between virulent PYS strain and avirulent PRU strain of Toxoplasma gondii. A, The distribution of phosphorylation on different residue types (pSer-serine, pThr-threonine, and pTyr-tyrosine) identified by TiO2. B, Distribution of single-, double-, triple- and quadruple- phosphorylated sites showed that the majority of phosphorylated peptides have only single phosphorylation site. C, Distribution of phosphorylated peptides matched by single spectrum 
and multiple spectra. The majority of phosphorylated peptides were matched by single spectrum. D, The numbers of phosphorylated sites on the identified phosphorylated proteins. The number of phosphorylated proteins with several phosphorylated sites decreases with the increase in the number of the phosphorylated sites.

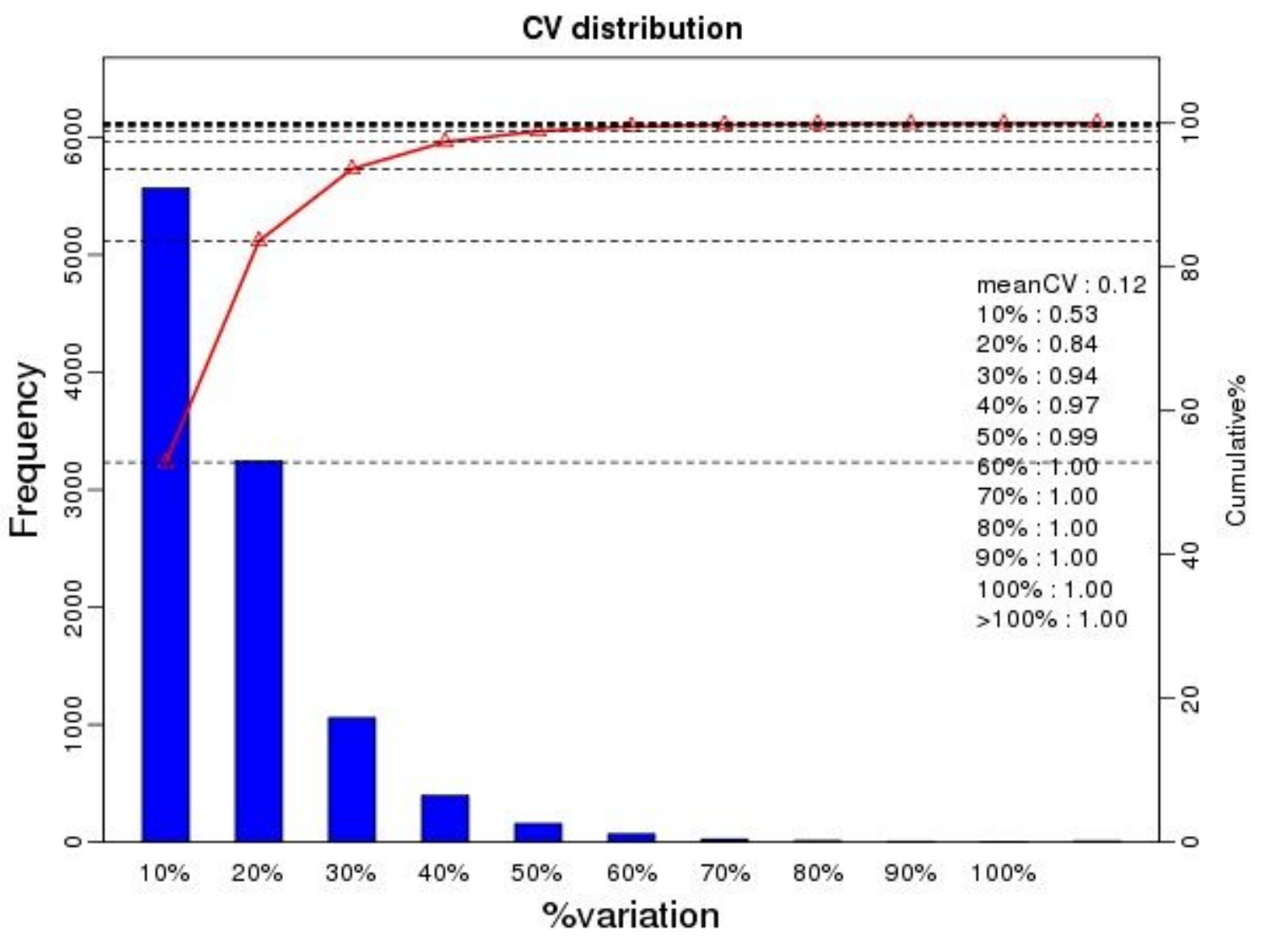

Figure 2

Reproducibility evaluation of phosphorylated peptides based on the value of Coefficient of variation (CV). The values of $\mathrm{CV}$ were indicated by percentage on the $\mathrm{X}$-axis. The left and the right $\mathrm{Y}$-axes represent the number of phosphopeptides and the cumulative percentage of phosphopeptides, respectively. 
$\mathbf{a}$

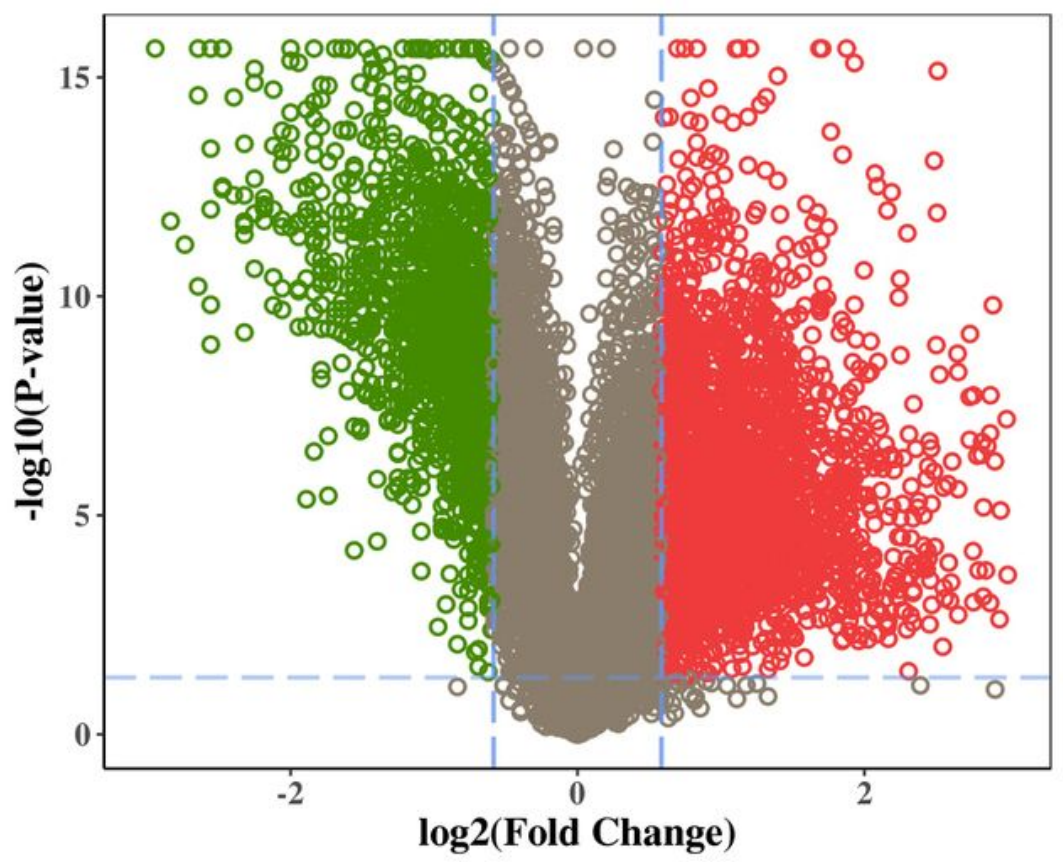

$\log 2$ (Fold Change)

2000

1500

1000

500

0

\section{1}

- Downregulated

- Unchanged

- Upregulated

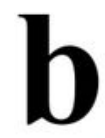


strain and PRU strain. The X-axis label represents values of log1.5 fold changes and the Y-axis label represents the quantity of phosphopeptides. Upregulated and downregulated DEPs were indicated by red and green colors, respectively.
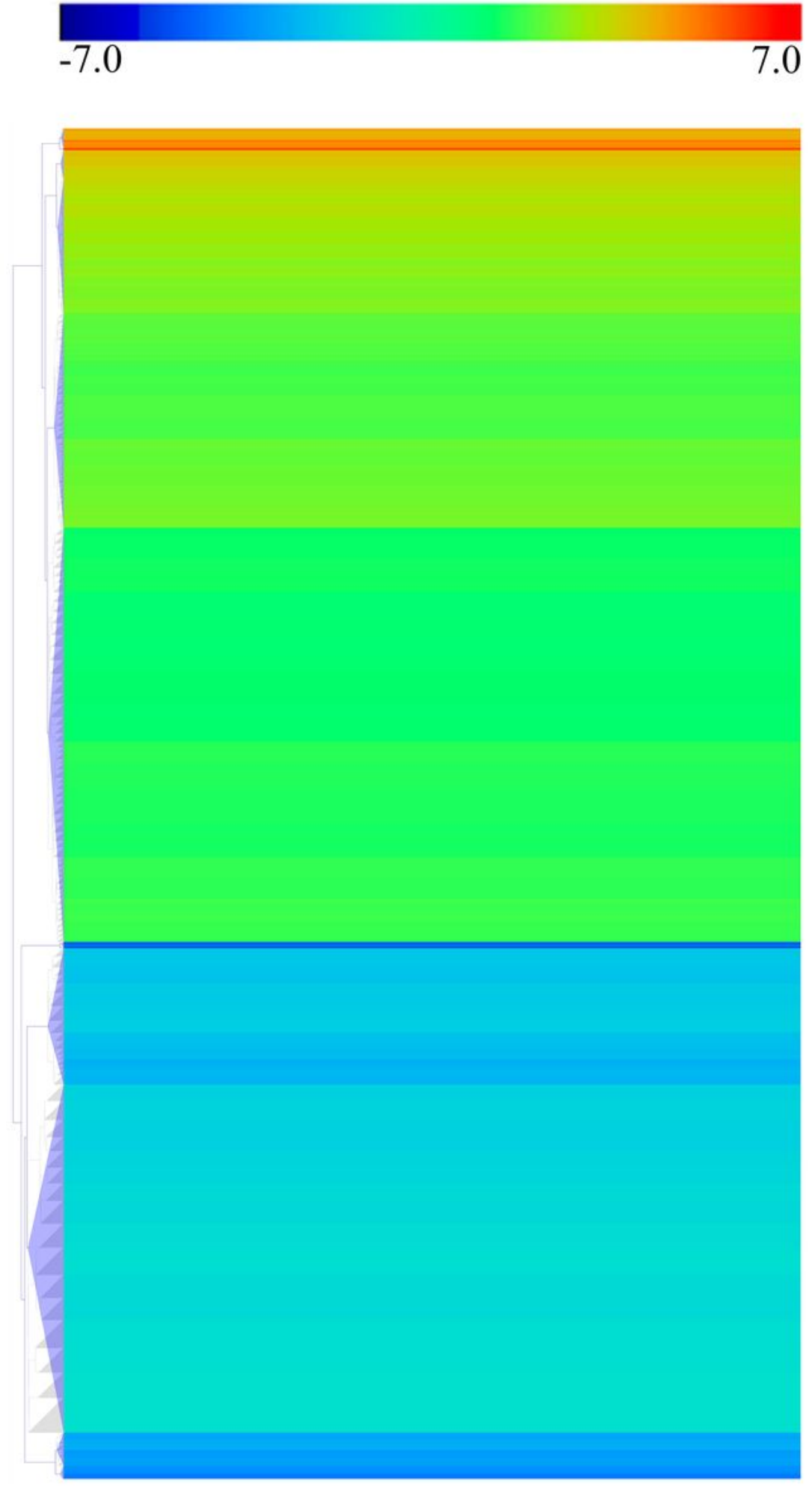

Figure 4

Hierarchical clustering heat map of differentially expressed phosphopeptides (DEPs). Expression values of DEPs were log1.5-transformed. 


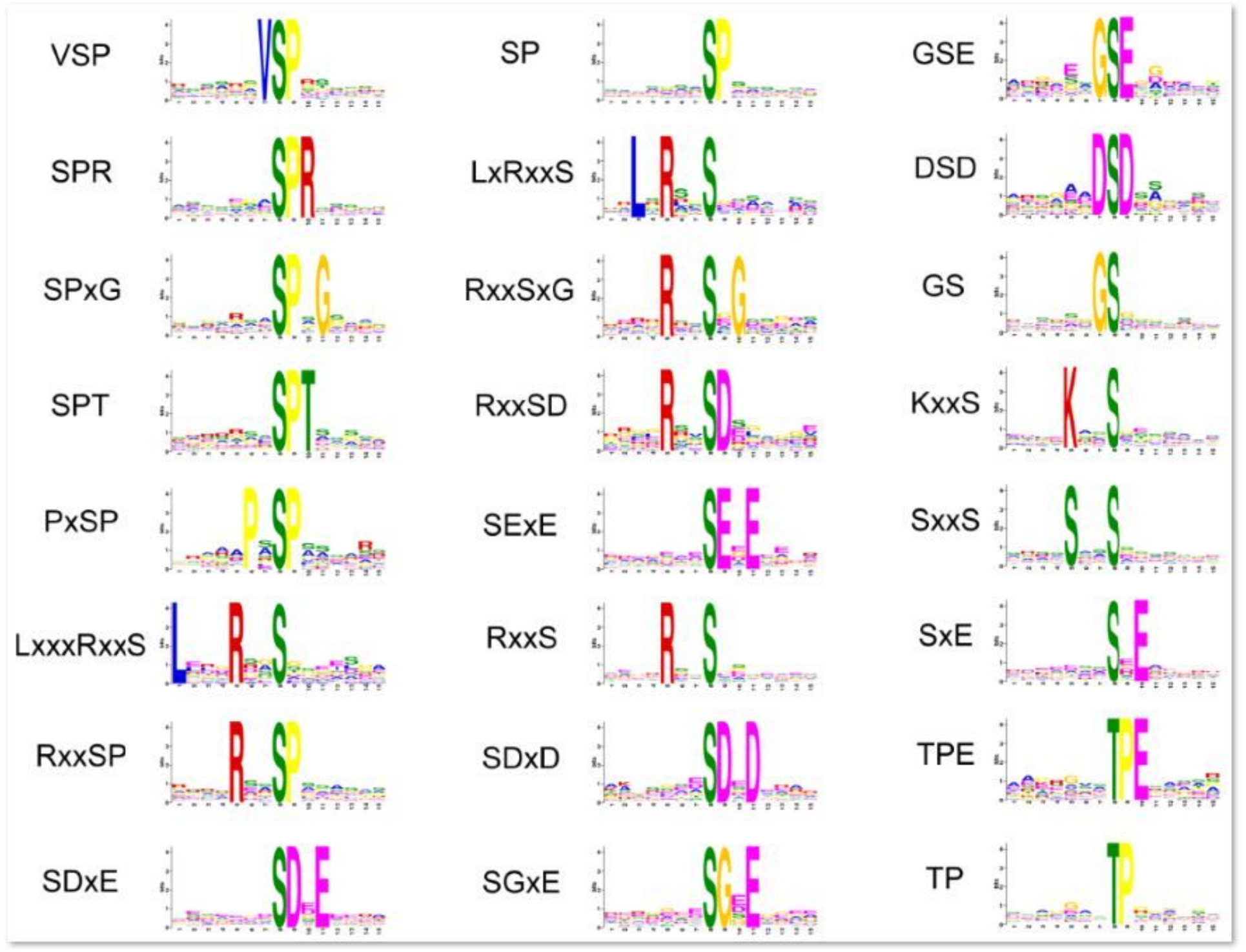

\section{Figure 5}

Identification of phosphorylation-specific motif within the upregulated DEPs using the Motif-X software. The frequency of amino acids appearing at specific positions were indicated by the height of the logos. The acidic and basic residues were represented in blue and red colors, respectively. 

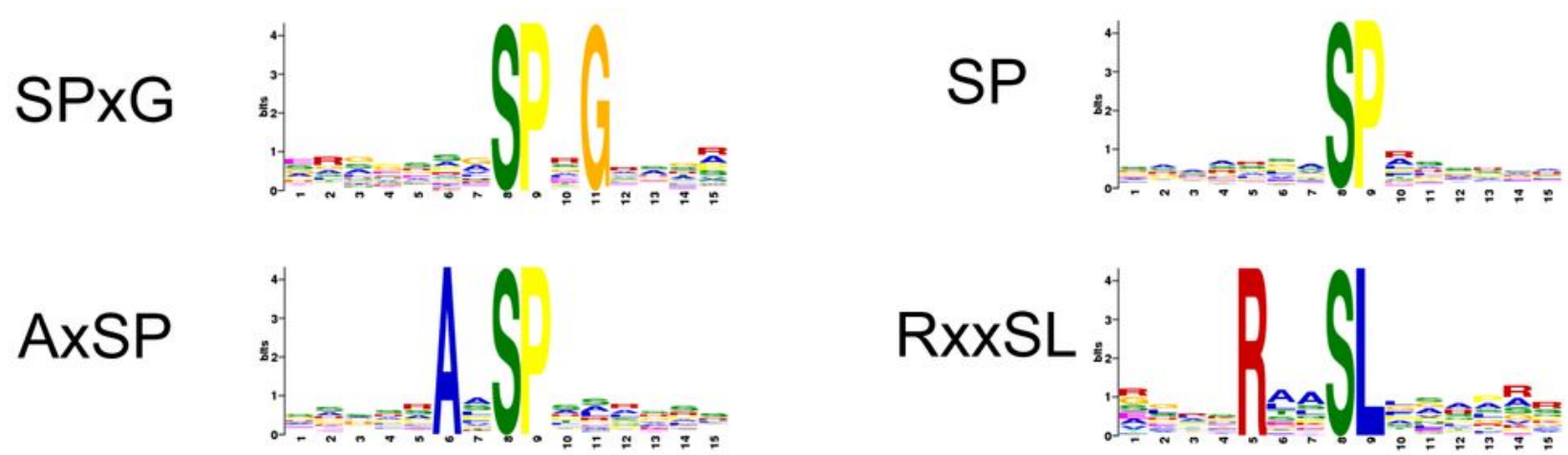

LSP
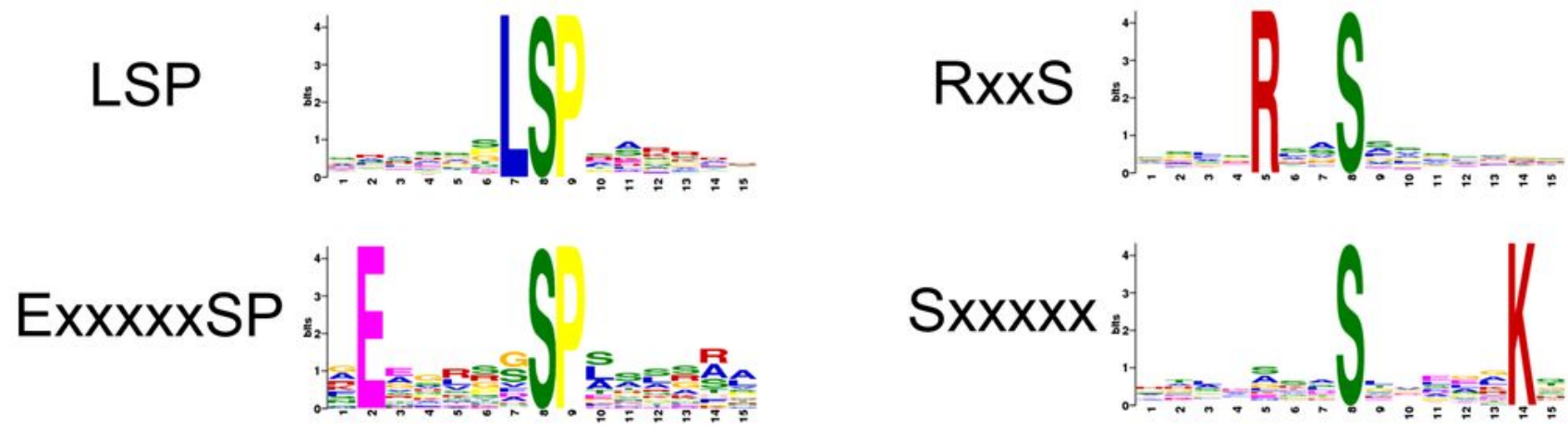

DxSP
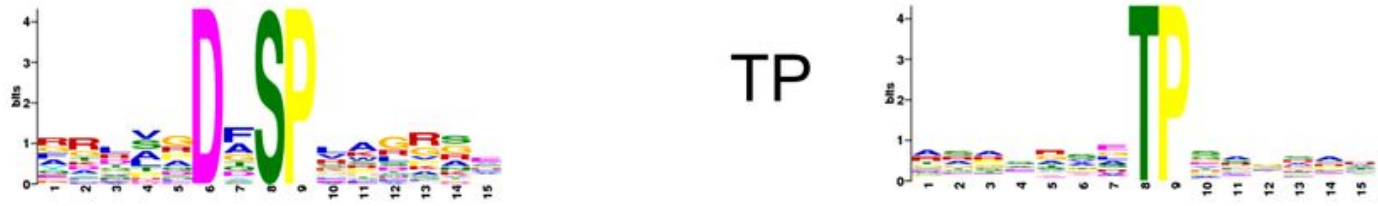

SPG
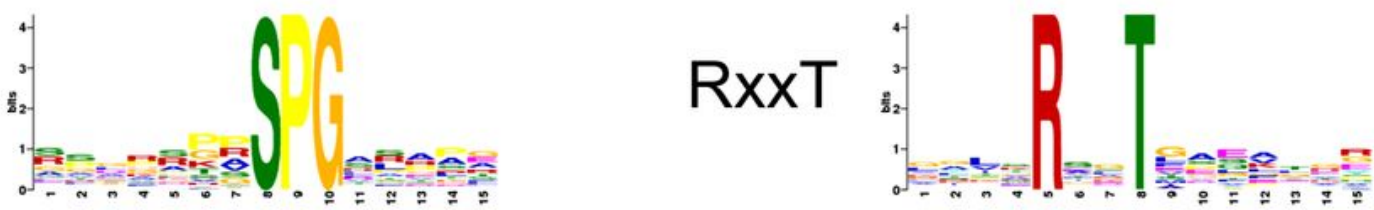

SPS
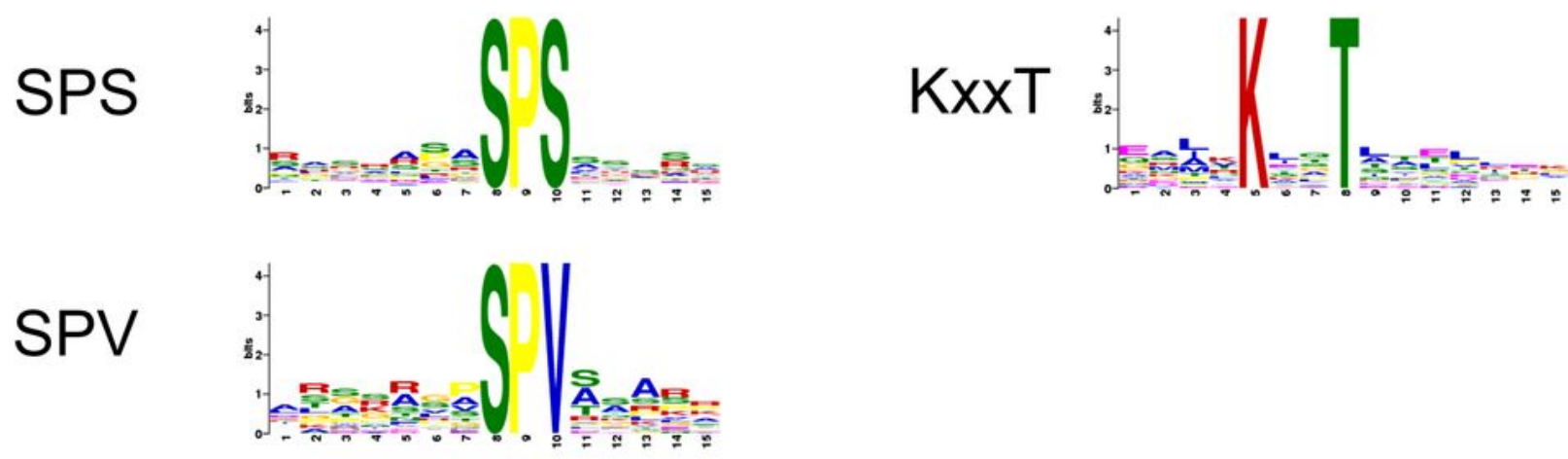

Figure 6

Identification of phosphorylation-specific motif within the downregulated DEPs using Motif-X software. The frequency of amino acids appearing at specific positions were indicated by the height of the logos. The acidic and basic residues were represented in blue and red colors, respectively. 
a

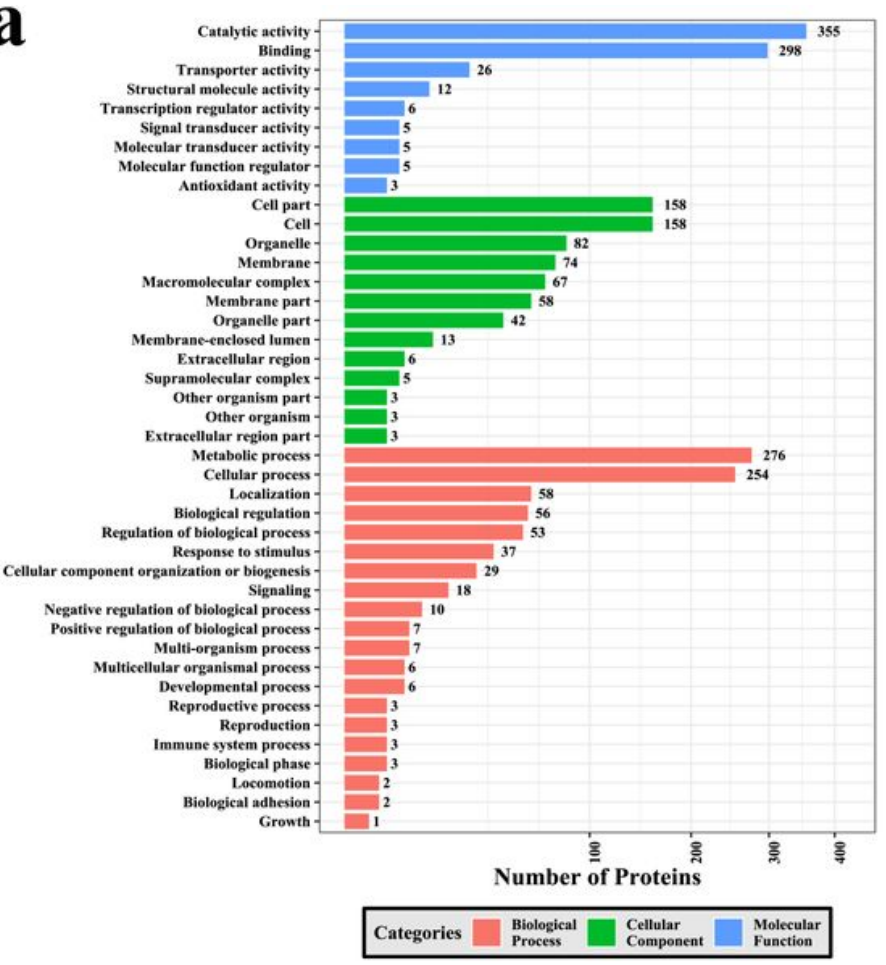

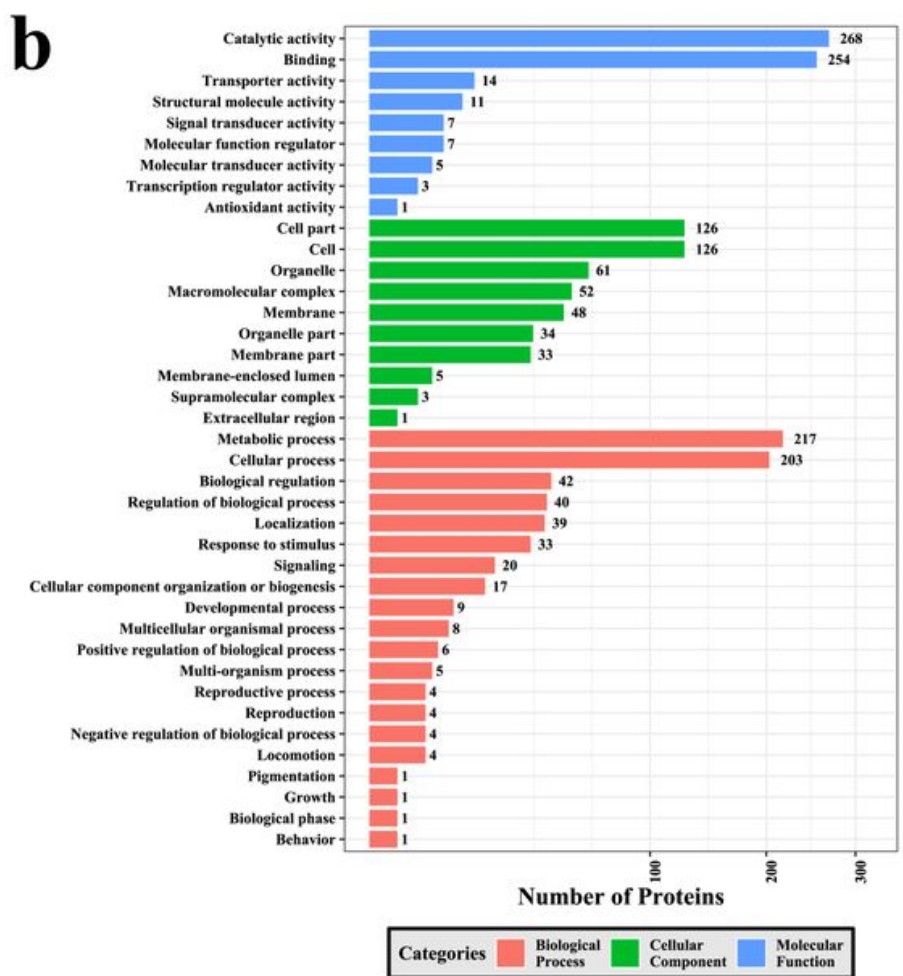

Figure 7

GO analysis of differentially expressed phosphoproteins (DEPs). The X-axis label represents the number of DEPs. The Y-axis label indicates $\mathrm{GO}$ terms of (A) upregulated and (B) downregulated phosphoproteins in PYS vs. PRU, respectively. 


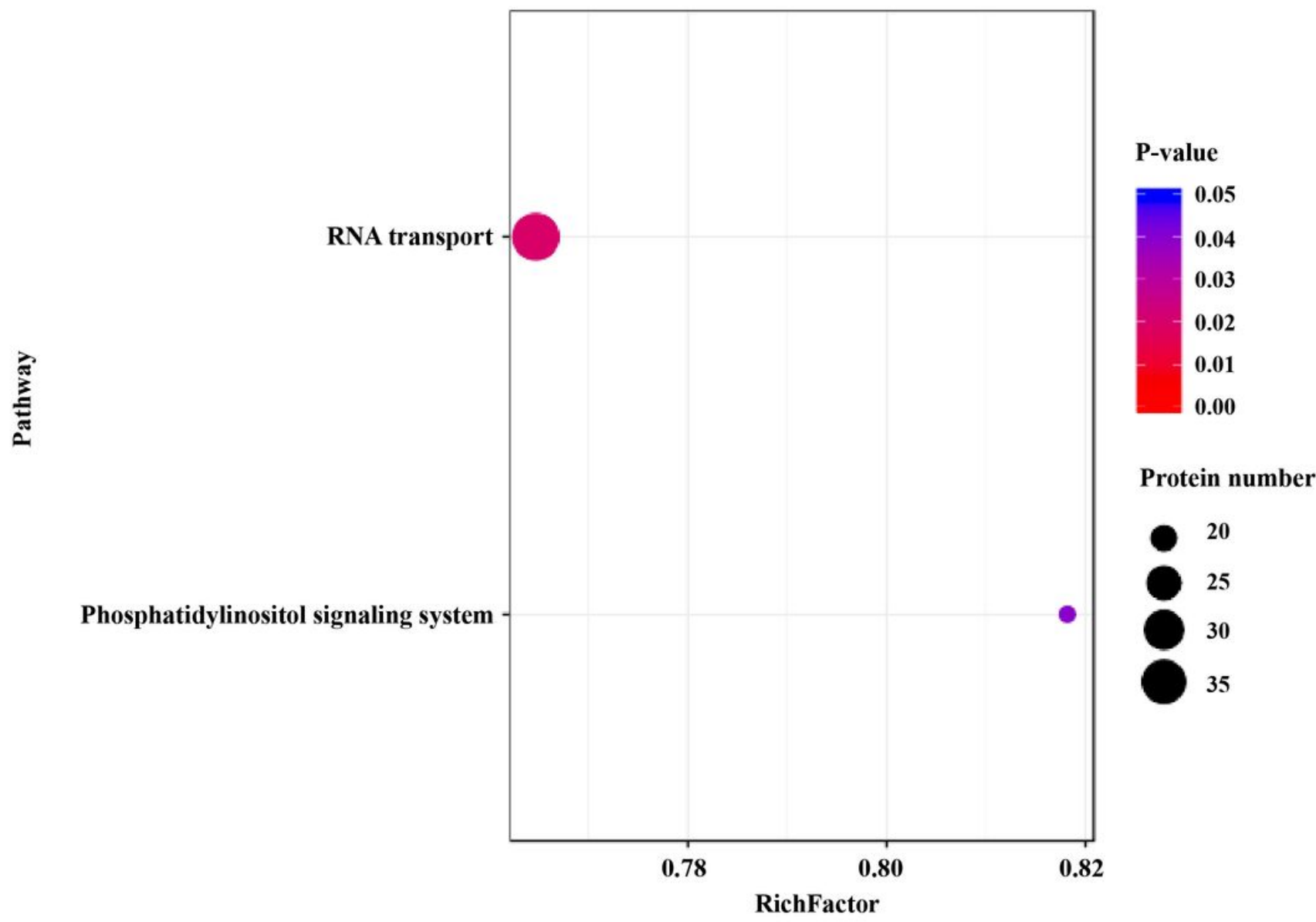

Figure 8

KEGG pathway enrichment analysis of differentially expressed phosphoproteins (DEPs) in PYS vs. PRU. The $Y$-axis label represents the significantly enriched KEGG pathways and the X-axis label shows the rich factor. The rich factor indicates the number of DEPs to the number of total phosphoproteins in the pathway. Higher rich factors indicate higher enrichment. The $p$ value and number of phosphoproteins in the pathways correspond to the color and size of the node, respectively. 


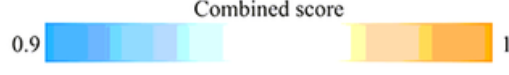

\section{DNA replication or RNA transcription}

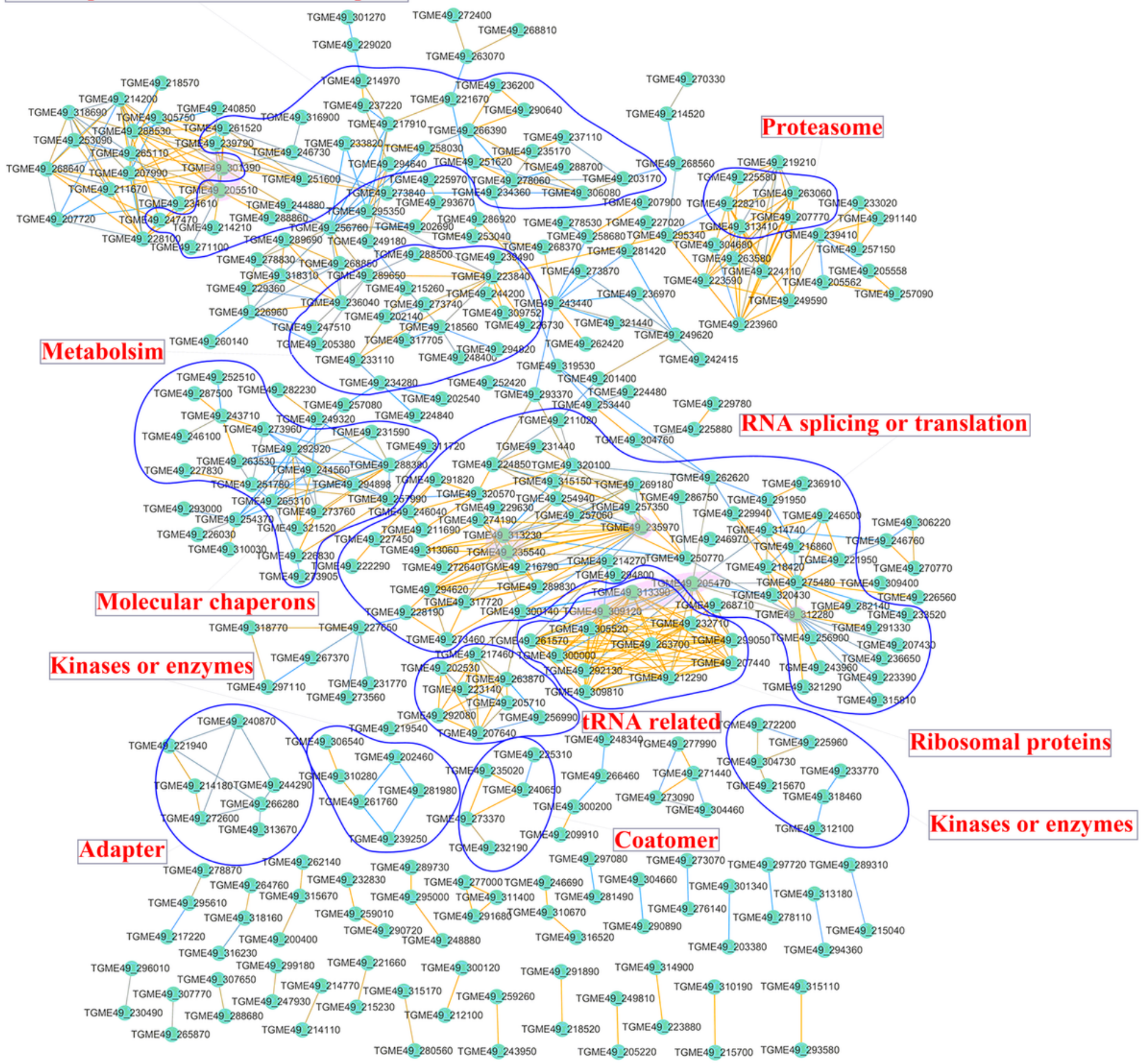

\section{Figure 9}

Phosphoprotein interaction network (PPI, combined score $\geq 0.9$ ) of the DEPs between PYS strain and PRU strain. The nodes denote DEPs and the edges represent interactors between DEPs. The color of edge represents the combined score of interactors. 


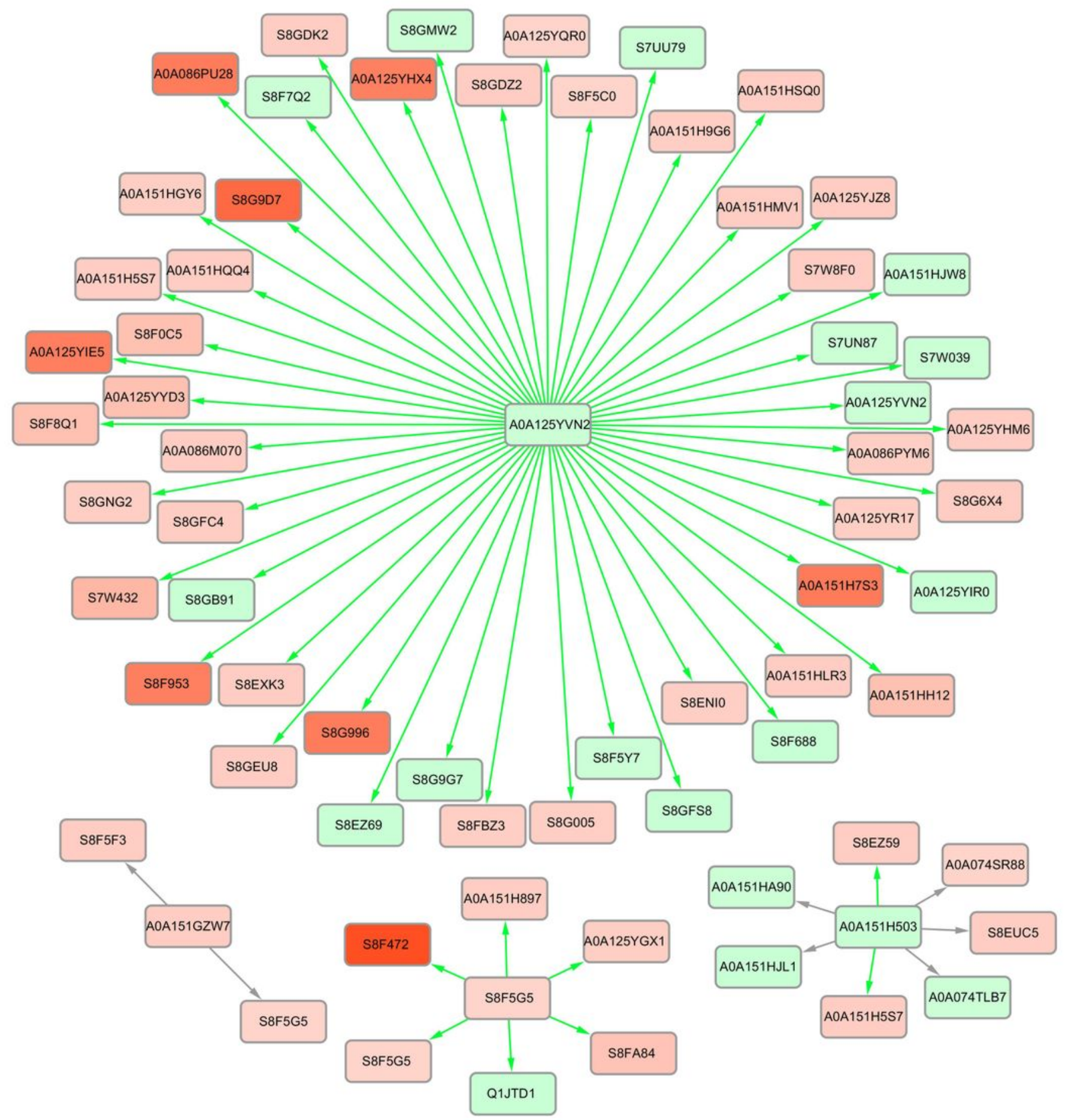

\section{Figure 10}

Network of kinase-phosphopeptide associations and potential phosphor-dependent interactions identified by correlation analysis. Log1.5(fold changes) of phosphoproteins upregulated/downregulated in PYS when comparing PYS/PRU were mapped in the nodes in red and green, respectively. The gray arrow indicates protein interactions that are predicted to be disabled by the phosphorylation and green arrows indicate protein interactions that are predicted to be enabled by the phosphorylation. 


\section{Supplementary Files}

This is a list of supplementary files associated with this preprint. Click to download.

- TableS1.xlsx

- TableS2.xIsx

- FigureS1.tif

- FigureS2.tif

- TableS3.xIsx

- TableS4.xlsx 